\title{
Crystalline nodal topological superconductivity and Bogolyubov Fermi surfaces in monolayer $\mathrm{NbSe}_{2}$
}

\author{
Daniel Shaffer, ${ }^{1}$ Jian Kang, ${ }^{2}$ F. J. Burnell, ${ }^{1}$ and Rafael M. Fernandes ${ }^{1}$ \\ ${ }^{1}$ School of Physics and Astronomy, University of Minnesota, Minneapolis, Minnesota 55455, USA \\ ${ }^{2}$ National High Magnetic Field Laboratory, Florida State University, Tallahassee, Florida 32310, USA
}

(Dated: May 14, 2020)

\begin{abstract}
We present a microscopic calculation of the phase diagram of the Ising superconductor $\mathrm{NbSe}_{2}$ in presence of both in-plane magnetic field and Rashba spin-orbit coupling (SOC). Repulsive interactions lead to two distinct instabilities, in singlet- and triplet- interaction channels. While we recover the previously predicted nodal topological superconducting state in the absence of Rashba SOC at large magnetic field with six pairs of nodes along $\Gamma-M$ lines, a finite Rashba SOC breaks the symmetry that protects these nodes and therefore generally lifts them, resulting in a topologically trivial phase. There is an exception when the field is applied along one of the three $\Gamma-K$ lines, however. In that case, a single mirror symmetry remains that can protect two pairs of nodes out of the original six, resulting in a crystalline topological superconducting phase. Depending on the Cooper pairs' center-of-mass momentum, this superconducting state displays either Bogolyubov Fermi surfaces or point nodes. Moreover, a chiral topological superconducting phase with Chern number of 6 is realized in the regime of large Rashba SOC and dominant triplet interactions, spontaneously breaking time-reversal symmetry.
\end{abstract}

\section{INTRODUCTION}

The observation of superconductivity (SC) in $1 \mathrm{H}$ monolayer transition metal dichalcogenides such as $\mathrm{NbSe}_{2}$ and $\mathrm{MoS}_{2}$ opens a new avenue to explore superconductivity in systems with strongly coupled spinorbital degrees of freedom [1-11. In contrast to their bulk counterparts, inversion symmetry is broken in these monolayers, giving rise to an Ising spin-orbit coupling (SOC) that forces the spins to point out-of-plane [7, 8, 12 14. This Ising SOC is believed to be responsible for the experimental observation that the superconducting state survives up to remarkably large in-plane magnetic fields, far beyond the usual Pauli limit [5, 7, 10, 15, 16].

The combination of large Ising SOC, which lifts the spin degeneracy, with multiple Fermi pockets has inspired considerable interest in the potential for unconventional superconductivity in these materials [12, 15, 17, 27]. In gated $\mathrm{MoS}_{2}$, which has four spin-split Fermi pockets centered at the $\pm K$ points of the hexagonal Brillouin zone, repulsive inter-band interactions can stabilize a fully gapped triplet SC state [19, 24, 25]. Chiral topological superconductivity [28] both with and without large Rashba SOC has also been predicted in $\mathrm{MoS}_{2}$ 24, 26, 29, as has finite-momentum Cooper pairing [26, 30. In $\mathrm{NbSe}_{2}$ and its close relative $\mathrm{TaS}_{2}$, which have Fermi pockets centered at the $\pm K$ and $\Gamma$ points, it was argued that for in-plane magnetic fields larger than the Pauli limiting field, a nodal topological SC state is realized, protected by an anti-unitary time-reversal like symmetry and characterized by Majorana flat bands at the sample's edges [17, 22].

Despite the flurry of activity on this front, important questions about the microscopic mechanism of unconventional SC and its stability in realistic experimental conditions remain unaddressed. Here, we go beyond phe-
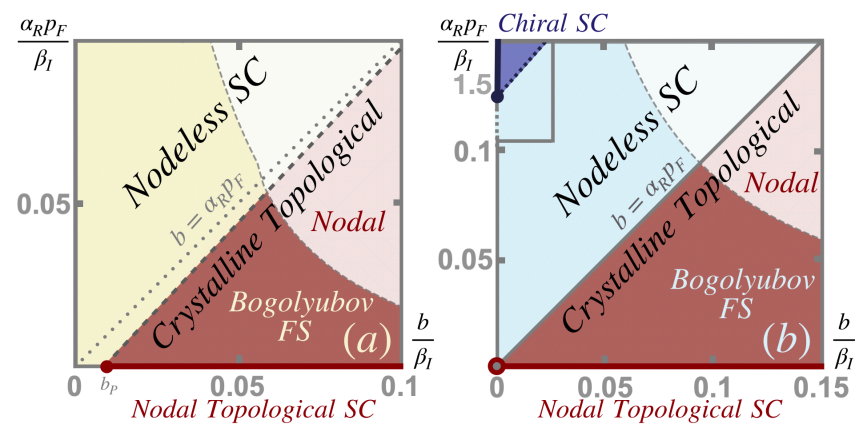

FIG. 1. Phase diagram for $\mathrm{NbSe}_{2}$ as a function of the Rashba SOC $\left(\alpha_{R}\right)$ and in-plane magnetic field $\mathbf{B}$ oriented along the $\Gamma-K$ direction, in units of the Ising SOC $\beta_{I}$. The leading SC instability at $\alpha_{R}=B=0$ is a singlet extended $s$-wave state or triplet $f$-wave state in panel (a) and (b) respectively. Solid (dashed) lines indicate exact (approximate) phase boundaries. Uniform SC becomes unstable in the light-shaded regions, but finite-momentum pairing remains possible. We discuss the phases in more detail in Sec. IVB We use parameter values given in Eqs. 8 and 45

nomenological models and present a microscopic theory of superconductivity in $\mathrm{NbSe}_{2}$ that considers the most relevant repulsive electronic interactions involving lowenergy fermions. Moreover, we include the simultaneous effects of an in-plane magnetic field $\mathbf{B}$ and Rashba SOC, with energy scale $\alpha_{R} p_{F}$ ( $p_{F}$ is the Fermi momentum). The latter is commonly present experimentally and can in principle be controlled by gating or by the choice of substrate. Importantly, it qualitatively changes the phase diagram: the nodal SC topological phase present at large fields [17] is generally destroyed by even a small Rashba SOC, as it lifts the nodes and breaks the time-reversallike symmetry protecting them. The exception is when the $\mathbf{B}$ field is parallel to one of the $\Gamma-K$ directions: in that 
case, nodes located along the direction perpendicular to $\mathbf{B}$ are protected by mirror symmetry, resulting in a crystalline topological SC phase that can be either nodal or exhibit protected Bogolyubov Fermi surfaces, depending on the momentum of the Cooper pairs.

Our analysis reveals two distinct $\left(B, \alpha_{R}\right)$ phase diagrams, shown in Fig. 1. For repulsive interactions, if the inter-band repulsion coupling the $\Gamma$ and $\pm K$ Fermi pockets dominates, the SC state for $B=\alpha_{R}=0$ is predominantly a singlet extended $s$-wave state with nearly isotropic gaps of opposite signs at $\Gamma$ and $\pm K$ (Fig. 1 1a). If the inter-band processes coupling the $K$ and $-K$ Fermi pockets dominates, the dominant SC instability for $B=\alpha_{R}=0$ is towards a triplet $f$-wave state, characterized by isotropic gaps of opposite signs at $K$ and $-K$, and a nodal gap at $\Gamma$. While the crystalline topological SC phase is present in both phase diagrams for large enough fields, a distinct chiral topological $p \pm i p$ SC that spontaneously breaks time-reversal symmetry is present for large $\alpha_{R}$ and $B \simeq 0$ in the phase diagram of Fig. 1(b).

The rest of the paper is organized as follows. In Sec. II we introduce our microscopic model, that includes Ising and Rashba SOC, in-plane magnetic field, and all symmetry allowed spin-conserving interactions. We analyze these interactions using a renormalization group (RG) analysis in Sec. III, and find that (at energy scales where SOC and the magnetic field can be neglected) superconductivity is the only instability. As a result, the RG at lower energy scales becomes equivalent to a selfconsistent mean field analysis. In Sec. IV we use this approach to study the superconducting phase diagram in the presence of SOC and magnetic field, and identify a new crystalline gapless topological superconducting phase at large in-plane magnetic field. Because the Fermi surfaces are no longer symmetric under momentum inversion $\mathbf{p} \rightarrow-\mathbf{p}$ in the presence of both magnetic field and Rashba SOC, we also calculate the boundary of the region where uniform superconductivity becomes unstable in Sec. IV C. We show in Sec. V that the gapless phase identified in Sec. IV] is a crystalline gapless topological phase, and analyze the resulting boundary modes using a simple tight binding model. We conclude in Sec. VI by discussing possible experimental signatures of the possible topological superconducting phases. A detailed discussion of the chiral state, as well as other technical supporting material, can be found in the appendices.

\section{MICROSCOPIC MODEL}

The Fermi surface of undoped $\mathrm{NbSe}_{2}$ is shown in Fig. 2. The non-interacting Hamiltonian is given by:

$$
H_{0}=\sum_{\eta \mathbf{p}} \psi_{\eta, \mathbf{p}}^{\dagger}\left[\epsilon_{\eta}(\mathbf{p})+\beta_{\eta}(\mathbf{p}) \sigma^{z}+\alpha_{R}(\boldsymbol{\sigma} \times \mathbf{p})_{z}\right] \psi_{\eta, \mathbf{p}}
$$

where $\psi_{\eta, \mathbf{p}}^{\dagger}=\left(d_{\eta, \mathbf{p} \uparrow}^{\dagger}, d_{\eta, \mathbf{p} \downarrow}^{\dagger}\right)$ and $d_{\eta, \mathbf{p} \alpha}^{\dagger}$ creates an electron at the pocket $\eta$ with $\operatorname{spin} \alpha=\uparrow, \downarrow$ and momentum p measured relative to the center of the pocket. The Fermi surface has three pairs of spin-split hole pockets centered at the $\pm K, \Gamma$ points in the Brillouin zone, which we label by $\eta= \pm K, \Gamma$, using the convention that $-\Gamma \equiv \Gamma$. Here $\epsilon_{\eta}(\mathbf{p})=-\frac{p^{2}}{2 m_{\eta}}-\mu$ is the band dispersion, with $m_{K}=$ $m_{-K}$. The Ising SOC has the form $\beta_{ \pm K}= \pm \beta_{I}$ near the $\pm K$ points and $\beta_{\Gamma}=2 \lambda p^{3} \cos 3 \theta$ near the $\Gamma$ point, where $\theta$ is the angle measured relative to the $\Gamma-K$ direction (see Fig. 2). Although Ising SOC vanishes along the $\Gamma-M$ lines, $\alpha_{R}$ does not, so the spin-degeneracy is fully lifted on all Fermi pockets. An in-plane magnetic field $\mathbf{B}$ adds a term $H_{\text {Zeeman }}=-\sum_{\eta \mathbf{p}} \psi_{\eta, \mathbf{p}}^{\dagger}(\mathbf{b} \cdot \boldsymbol{\sigma}) \psi_{\eta, \mathbf{p}}$, where $\mathbf{b} \equiv \frac{1}{2} g_{L} \mu_{B} \mathbf{B}$ and $g_{L}$ is the Landé g-factor; this also lifts the spin-degeneracy along the $\Gamma-M$ lines. We then have

$$
H_{0}+H_{\text {Zeeman }}=\sum_{\eta \mathbf{p}} \psi_{\eta, \mathbf{p}}^{\dagger}\left[\epsilon_{\eta}(\mathbf{p})+\boldsymbol{\delta}_{\eta}(\mathbf{p}) \cdot \boldsymbol{\sigma}\right] \psi_{\eta, \mathbf{p}}
$$

where

$$
\boldsymbol{\delta}_{\eta}(\mathbf{p})=\beta_{\eta}(\mathbf{p}) \hat{\mathbf{z}}+\alpha_{R}\left(p_{y} \hat{\mathbf{x}}-p_{x} \hat{\mathbf{y}}\right)+\mathbf{b}
$$

is the effective magnetic field seen by an electron with momentum p. The eigenvalues of the non-interacting Hamiltonian are therefore given by

$$
\xi_{\eta \tau}(\mathbf{p})=\epsilon_{\eta}(\mathbf{p})+\tau \delta_{\eta}(\mathbf{p})
$$

where $\delta_{\eta}=\left|\boldsymbol{\delta}_{\eta}\right|$, and the eigenstates are related to the electron annihilation operators $d_{\eta, \mathbf{p} \alpha}$ by the unitary transformation

$$
c_{\eta, \mathbf{p} \tau}=U_{\eta \tau}^{\alpha}(\mathbf{p}) d_{\eta, \mathbf{p} \alpha}
$$

Here $\tau=+1(-1)$ on the outer (inner) spin-split Fermi surface, $\alpha=1(-1)$ for spin up (spin down) (see Fig. 2), and we have defined

$$
\begin{aligned}
U_{\Gamma \tau}^{\alpha}(\mathbf{p}) & =\sqrt{\frac{\delta_{\Gamma}+\tau \alpha\left(2 \lambda p^{3} \cos 3 \theta_{\mathbf{p}}\right)}{2 \delta_{\Gamma}}}\left(\tau e^{-i \phi}\right)^{\frac{1+\alpha}{2}} \\
U_{ \pm K \tau}^{\alpha}(\mathbf{p}) & =\sqrt{\frac{\delta_{K} \pm \tau \alpha \beta_{I}}{2 \delta_{K}}}\left(\tau e^{-i \phi}\right)^{\frac{1+\alpha}{2}}
\end{aligned}
$$

where

$$
\begin{aligned}
\delta_{\Gamma} & =\sqrt{\left(2 \lambda p^{3} \cos (3 \theta)\right)^{2}+\left(\alpha_{R} p_{y}+b_{x}\right)^{2}+\left(\alpha_{R} p_{x}-b_{y}\right)^{2}} \\
\delta_{K} & =\sqrt{\beta_{I}^{2}+\left(\alpha_{R} p_{y}+b_{x}\right)^{2}+\left(\alpha_{R} p_{x}-b_{y}\right)^{2}} \\
e^{i \phi} & =\frac{\alpha_{R} p_{y}+b_{x}+i\left(-\alpha_{R} p_{x}+b_{y}\right)}{\sqrt{\left(\alpha_{R} p_{y}+b_{x}\right)^{2}+\left(\alpha_{R} p_{x}-b_{y}\right)^{2}}}
\end{aligned}
$$

Experimentally, the Ising SOC at $K$ points $\beta_{I}$ is found to be much smaller than the bandwidth. Thus in the weak coupling theory used here, the overall energy scale is set by $\beta_{I}$, and the momentum scale is set by the Fermi momentum $p_{F}$. To produce the plots in this paper, we took equal masses and chemical potentials at $\Gamma$ and $K$ points, and chose

$$
m=1.5 \frac{p_{F}^{2}}{\beta_{I}}, \quad \mu=-5 \beta_{I}, \quad \lambda=0.5 \beta_{I}
$$




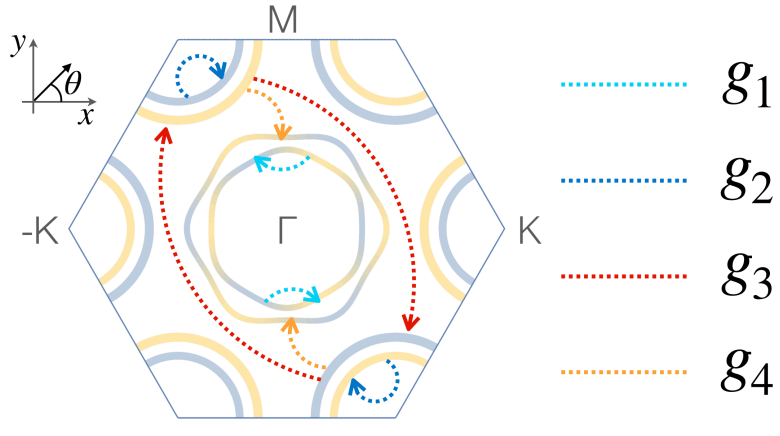

FIG. 2. The Fermi surface of $\mathrm{NbSe}_{2}$ in the presence of Ising SOC and a weak Rashba SOC. The colors indicate the outof-plane spin polarization of each pocket. The arrows denote the four distinct types of repulsive electronic interactions that contribute to the pairing instability. We used parameter values in Eq. 8 for the non-interacting Hamiltonian, with $b=0.5 \beta_{I}$, and $\alpha_{R}=0$; the Fermi surfaces are independent of the direction of the magnetic field in this case $(b=0$, and $\alpha_{R} p_{F}=0.5 \beta_{I}$ give the same Fermi surface). We picked values somewhat larger than reported in experiment to produce visible splitting at the $\Gamma$ pocket.

with $p$ shown in units of $p_{F}$. This choice roughly matches the Fermi surfaces reported in 17, 10, 17, 20, Experimentally, for bulk $\mathrm{NbSe}_{2}, p_{F} / \hbar=0.5 \AA^{-1} 31,32$. Note that the estimated value of $\beta_{I}$ is of the order of $40 \mathrm{meV}$ [7, 10. Thus, the maximum values of $b$ in our phase diagrams of Fig. 1 correspond roughly to fields of $70 \mathrm{~T}$ and $100 \mathrm{~T}$. Although the largest values in this range are most likely above the experimental critical in-plane field value, given the uncertainty in the microscopic parameters, we opt to extend the phase diagrams across a wide range of magnetic field values.

\section{Interactions Near the Fermi Surfaces}

Symmetry constrains the possible spin-conserving, momentum-direction-independent electronic interactions between the low-energy fermionic operators to eight. Of these, the four interactions that contribute to superconductivity are (see Fig. 2): intra-pocket density-density interactions involving the $\Gamma\left(g_{1}\right)$ and the $\pm K\left(g_{2}\right)$ pockets; and inter-pocket pair-hopping interactions between $K$ and $-K\left(g_{3}\right)$ and between $\Gamma$ and $\pm K\left(g_{4}\right)$. Thus we consider the following interacting Hamiltonian:

$$
\begin{aligned}
H_{\text {Int }}= & V_{\Gamma ; \Gamma}^{\alpha^{\prime} \beta^{\prime} ; \alpha \beta}(\mathbf{p} ; \mathbf{k}) d_{\Gamma, \mathbf{p} \alpha}^{\dagger} d_{\Gamma,-\mathbf{p} \beta^{\prime}}^{\dagger} d_{\Gamma, \mathbf{k} \alpha^{\prime}} d_{\Gamma,-\mathbf{k} \beta^{\prime}}+ \\
& V_{ \pm K ; \pm K}^{\alpha^{\prime} \beta^{\prime} ; \alpha \beta}(\mathbf{p} ; \mathbf{k}) d_{ \pm K, \mathbf{p} \alpha}^{\dagger} d_{\mp K,-\mathbf{p} \beta}^{\dagger} d_{ \pm K, \mathbf{k} \alpha^{\prime}} d_{\mp K,-\mathbf{k} \beta^{\prime}}+ \\
& V_{ \pm K ; \mp K}^{\alpha^{\prime} \beta^{\prime} ; \alpha \beta}(\mathbf{p} ; \mathbf{k}) d_{ \pm K, \mathbf{p} \alpha}^{\dagger} d_{\mp K,-\mathbf{p} \beta}^{\dagger} d_{\mp K, \mathbf{k} \alpha^{\prime}} d_{ \pm K,-\mathbf{k} \beta^{\prime}}+ \\
& V_{\Gamma ; \pm K}^{\alpha^{\prime} \beta^{\prime} ; \alpha \beta}(\mathbf{p} ; \mathbf{k}) d_{ \pm K, \mathbf{p} \alpha}^{\dagger} d_{\mp K,-\mathbf{p} \beta}^{\dagger} d_{\Gamma, \mathbf{k} \alpha^{\prime}} d_{\Gamma,-\mathbf{k} \beta^{\prime}}+\text { h.c. }
\end{aligned}
$$

Accounting for the anti-symmetric nature of the fermion operators (and including all Hermitian conjugates), the uniform part of the interactions can be separated into singlet and triplet interaction channels, as follows:

$$
\begin{aligned}
{\left[V^{s}\right]_{\Gamma ; \Gamma}^{\alpha^{\prime} \beta^{\prime} ; \alpha \beta} } & =\frac{g_{1}}{2}\left(i \sigma^{y}\right)^{\alpha \beta}\left(i \sigma^{y}\right)^{\alpha^{\prime} \beta^{\prime}} \\
{\left[V^{s}\right]_{\Gamma ; \pm K}^{\alpha^{\prime} \beta^{\prime} ; \alpha \beta} } & = \pm \frac{g_{4}}{2}\left(i \sigma^{y}\right)^{\alpha \beta}\left(i \sigma^{y}\right)^{\alpha^{\prime} \beta^{\prime}} \\
{\left[V^{s}\right]_{ \pm K ; \pm K}^{\alpha^{\prime} \beta^{\prime} ; \alpha \beta} } & =\frac{1}{4}\left(g_{2}+g_{3}\right)\left(i \sigma^{y}\right)^{\alpha \beta}\left(i \sigma^{y}\right)^{\alpha^{\prime} \beta^{\prime}} \\
{\left[V^{t}\right]_{ \pm K ; \pm K}^{\alpha^{\prime} \beta^{\prime} ; \alpha \beta} } & =\frac{1}{4}\left(g_{2}-g_{3}\right) \sum_{j=x, y, z}\left(\sigma^{j} i \sigma^{y}\right)_{\alpha \beta}^{*}\left(\sigma^{j} i \sigma^{y}\right)^{\alpha^{\prime} \beta^{\prime}}
\end{aligned}
$$

Since $V_{K, K}$ and $V_{K,-K}$ are related by interchanging the spin indices $\alpha^{\prime}, \beta^{\prime}$, combined with an overall minus sign for interchanging two fermion operators, in this representation we have

$$
\begin{aligned}
{\left[V^{s}\right]_{ \pm K ; \mp K}^{\alpha^{\prime} \beta^{\prime} ; \alpha \beta} } & =\left[V^{s}\right]_{ \pm K ; \pm K}^{\alpha^{\prime} \beta^{\prime} ; \alpha \beta} \\
{\left[V^{t}\right]_{ \pm K ; \mp K}^{\alpha^{\prime} \beta^{\prime} ; \alpha \beta} } & =-\left[V^{t}\right]_{ \pm K ; \pm K}^{\alpha^{\prime} \beta^{\prime} ; \alpha \beta}
\end{aligned}
$$

From Eq. 10, , we see that $V_{ \pm K, \pm K}$ (and thus $\left.V_{ \pm K, \mp K}\right)$ have contributions in both the singlet channel (labeled $s$ ) and the triplet channel (labeled $t$ ), while for momentumdirection-independent interactions, $V_{\Gamma, \Gamma}$ and $V_{\Gamma, K}$ have contributions only in the singlet channel. In addition to these interactions, in order to ensure that the gap on the $\Gamma$ pocket does not artificially vanish in the triplet regime, we also include weak (but symmetry-allowed) momentum-direction dependent interactions:

$$
\begin{aligned}
& {\left[V^{t}(\mathbf{p} ; \mathbf{k})\right]_{\Gamma ; \Gamma}^{\alpha^{\prime} \beta^{\prime} ; \alpha \beta}=\frac{g_{1}^{t}}{2} \cos 3 \theta_{\mathbf{k}} \cos 3 \theta_{\mathbf{p}}\left(\boldsymbol{\sigma} i \sigma^{y}\right)_{\alpha \beta}^{*} \cdot\left(\boldsymbol{\sigma} i \sigma^{y}\right)^{\alpha^{\prime} \beta^{\prime}}} \\
& {\left[V^{t}(\mathbf{p} ; \mathbf{k})\right]_{\Gamma ; \pm K}^{\alpha^{\prime} \beta^{\prime} ; \alpha \beta}= \pm \frac{g_{4}^{t}}{\sqrt{2}} \cos 3 \theta_{\mathbf{k}}\left(\boldsymbol{\sigma} i \sigma^{y}\right)_{\alpha \beta}^{*} \cdot\left(\boldsymbol{\sigma} i \sigma^{y}\right)^{\alpha^{\prime} \beta^{\prime}}}
\end{aligned}
$$

where $\theta_{\mathbf{k}}$ refers to the angle of the momentum on the $\Gamma$ pocket. Note that all higher harmonics can be included in a similar fashion, but they do not qualitatively affect our conclusions. We take $\left|g_{i}^{t}\right| \ll\left|g_{i}\right|$, so these interactions have a negligible effect on whether the system enters the singlet or triplet regime.

Note that there are four other possible interactions, which we do not include in our analysis here,

$$
\begin{aligned}
& H_{\text {Int }}=\frac{g_{5}}{2} d_{K \alpha}^{\dagger} d_{K \beta}^{\dagger} d_{K \beta} d_{K \alpha}+\frac{g_{6}}{2} d_{-K \alpha}^{\dagger} d_{\Gamma \beta}^{\dagger} d_{\Gamma \beta} d_{-K \alpha}+ \\
& +\frac{g_{7}}{2} d_{-K \alpha}^{\dagger} d_{\Gamma \beta}^{\dagger} d_{-K \beta} d_{\Gamma \alpha}+\frac{g_{8}}{2} d_{-K \alpha}^{\dagger} d_{\Gamma \beta}^{\dagger} d_{K \beta} d_{K \alpha}+\text { h.c. }
\end{aligned}
$$

where we omitted momentum indices and symmetry related terms for simplicity. These interactions decouple from $g_{1}, \ldots g_{4}$, and hence need not be included when analyzing the possible superconducting instabilities. They can in principle give rise to a pair density wave (PDW) which may compete with superconductivity, depending on the microscopic values of $g_{5}, \ldots g_{8}$. We defer analysis of this possibility to future work. 


\section{RENORMALIZATION GROUP AND SUPERCONDUCTING INSTABILITY}

To determine which instabilities are favored by the interactions above, we perform a parquet RG analysis, keeping only the dominant momentum-directionindependent interactions of Eq. (10). This approach is appropriate for the situation when the Fermi energy is small compared to the bandwidth, as one integrates out states from energies of the order of the bandwidth to energies of the order of the Fermi energy [33 36]; in the case of $\mathrm{NbSe}_{2}$ the Fermi surfaces can be made small by controlling the gate voltage. Below the Fermi energy, the different channels decouple, and in the absence of nesting, the only logarithmic instability is the superconducting one. Because the energy scale of the Ising SOC is smaller than the Fermi energy, we perform the parquet $\mathrm{RG}$ in the absence of SOC or magnetic fields. These terms however are relevant as we move to energy scales below the Fermi energy, which we explore in Section IV.

\section{A. RG Flow Equations}

It is convenient to rescale the coupling constants by the density of states (DOS) $N_{\eta}=\frac{m_{\eta}}{2 \pi}$ of the $\eta$ pocket (by symmetry $\left.N_{K}=N_{-K}\right)$ :

$$
\begin{array}{lll}
\tilde{g}_{1}^{(s)}=N_{\Gamma} g_{1}, & \tilde{g}_{4}^{(s)}=\sqrt{N_{\Gamma} N_{K}} g_{4}, & \tilde{g}_{23}^{(s)}=N_{K} \frac{g_{2}+g_{3}}{2} \\
\tilde{g}_{1}^{(t)}=N_{\Gamma} g_{1}^{t}, & \tilde{g}_{4}^{(t)}=\sqrt{N_{\Gamma} N_{K}} g_{4}^{t}, & \tilde{g}_{23}^{(t)}=N_{K} \frac{g_{2}-g_{3}}{2}
\end{array}
$$

We use the standard parquet RG procedure [33 36]. Since all pockets are hole pockets, only ladder diagrams contribute to logarithmic instabilities at one-loop order. In the absence of SOC the RG flow equations for for the singlet $(a=s)$ and triplet $(a=t)$ channels decouple:

$$
\begin{aligned}
& \dot{\tilde{g}}_{1}^{(a)}=-\left(\tilde{g}_{1}^{(a)}\right)^{2}-2\left(\tilde{g}_{4}^{(a)}\right)^{2} \\
& \dot{\tilde{g}}_{23}^{(a)}=-2\left(\tilde{g}_{23}^{(a)}\right)^{2}-\left(\tilde{g}_{4}^{(a)}\right)^{2} \\
& \dot{\tilde{g}}_{4}^{(a)}=-\left(\tilde{g}_{1}^{(a)}+2 \tilde{g}_{23}^{(a)}\right) \tilde{g}_{4}^{(a)}
\end{aligned}
$$

where the dot indicates a derivative with respect to the RG scale $s$ determined by the pairing susceptibility via

$-N_{\eta} s=\Pi_{\eta}=T \sum_{n} \int_{d \Lambda} G_{\eta}^{(0)}\left(i \omega_{n}, \mathbf{Q}\right) G_{-\eta}^{(0)}\left(-i \omega_{n},-\mathbf{Q}\right) \frac{d^{2} Q}{(2 \pi)^{3}}$

Here $\omega_{n}$ are Matsubara frequencies, and the momentum integral is restricted to a thin shell at energy $\Lambda$ and of thickness $d \Lambda \equiv \Lambda s . G_{\eta}^{(0)}\left(i \omega_{n}, \mathbf{Q}\right)=\left(i \omega_{n}-\epsilon_{\eta}(\mathbf{Q})\right)^{-1}$ is the bare normal state Green's function.

Eq. 15 has an analytic solution, which can be obtained by switching to a cylindrical coordinate system in the $\tilde{g}_{1}^{(a)}, \tilde{g}_{23}^{(a)}$, and $\tilde{g}_{4}^{(a)}$ parameter space:

$$
z^{(a)}=2 \tilde{g}_{23}^{(a)}+\tilde{g}_{1}^{(a)}
$$

$$
r^{(a)} \cos \theta^{(a)}=\tilde{g}_{4}^{(a)} \quad r^{(a)} \sin \theta^{(a)}=2 \tilde{g}_{23}^{(a)}-\tilde{g}_{1}^{(a)}
$$

Eq. 15 becomes:

$$
\begin{aligned}
& \dot{z}^{(a)}=-\frac{\left(z^{(a)}\right)^{2}}{2}-\frac{\left(r^{(a)}\right)^{2}}{2}\left(1+7 \cos ^{2} \theta^{(a)}\right) \\
& \dot{r}^{(a)}=-r^{(a)} z^{(a)} \\
& \dot{\theta}^{(a)}=0
\end{aligned}
$$

We see that one channel, parametrized by $\theta^{(a)}$, is not renormalized within one-loop. The other two channels decouple, according to:

$$
\dot{\gamma}^{(a \pm)}=\left(\gamma^{(a \pm)}\right)^{2}
$$

where, in terms of the coupling constants $\tilde{g}^{a}$, we have:

$$
\begin{aligned}
\gamma^{(a \pm)} & =-\frac{1}{2}\left(z^{(a)} \mp r^{(a)} \sqrt{1+7 \cos ^{2} \theta^{(a)}}\right) \\
& =-\frac{1}{2} \tilde{g}_{1}^{(a)}-\tilde{g}_{23}^{(a)} \pm \frac{1}{2} \sqrt{\left(\tilde{g}_{1}^{(a)}-2 \tilde{g}_{23}^{(a)}\right)^{2}+8\left(\tilde{g}_{4}^{(a)}\right)^{2}}
\end{aligned}
$$

When $\gamma^{(a \pm)}>0$, the associated coupling constant flows to $\infty$. Since $\gamma^{(a+)}>\gamma^{(a-)}$, the former always diverges faster than the latter, so we need only analyze the $\gamma^{(a+)}$ solution. When $N_{\eta}=N$ are all equal, these can be expressed:

$\gamma^{(s)}=\gamma^{(s+)}=-\frac{N}{2}\left(g_{1}+g_{2}+g_{3}\right)+\frac{N}{2} \sqrt{\left(g_{1}-g_{2}-g_{3}\right)^{2}+8 g_{4}^{2}}$

in the singlet channel and

$$
\gamma^{(t)}=\gamma^{(t+)}=\frac{N}{2}\left(g_{3}-g_{2}+\left|g_{2}-g_{3}\right|\right)
$$

in the triplet channel.

\section{B. Superconducting Instability}

Having determined the RG flow of the coupling constants, we now discuss which instabilities they cause. To do so, we introduce vertices associated with different types of electronic order $\left(\Delta_{\Gamma}(\mathbf{p})\right)_{\alpha \beta} \propto\left\langle d_{\Gamma, \mathbf{p} \alpha} d_{\Gamma,-\mathbf{p} \beta}\right\rangle$ at the $\Gamma$ pocket, and $\left(\Delta_{ \pm K}(\mathbf{p})\right)_{\alpha \beta} \propto\left\langle d_{ \pm K, \mathbf{p} \alpha} d_{\mp K,-\mathbf{p} \beta}\right\rangle$ at the $\pm K$ pockets. Here $\alpha \beta$ are spin indices. In the absence of SOC the corresponding superconducting pairing vertices can be decomposed into singlet and triplet channels as follows:

$$
\begin{aligned}
\Delta_{\eta}^{(s)}(\mathbf{p}) & =D_{\eta}^{(s)} i \sigma^{y} \\
\Delta_{\Gamma}^{(t)}(\mathbf{p}) & =D_{\Gamma}^{(t)} \sqrt{2} \cos 3 \theta_{\Gamma, \mathbf{p}} \hat{\mathbf{d}} \cdot \boldsymbol{\sigma} i \sigma^{y} \\
\Delta_{ \pm K}^{(t)}(\mathbf{p}) & = \pm D_{K}^{(t)} \hat{\mathbf{d}} \cdot \boldsymbol{\sigma} i \sigma^{y}
\end{aligned}
$$

where $\theta_{\Gamma, \mathbf{p}}$ is the angle about the $\Gamma$ Fermi surface, $D_{\eta}^{(a)}$ are momentum-independent coefficients, and the (unit) $\hat{\mathbf{d}}$ vector is the same on $\Gamma$ and $K$ pockets. Note that 
$\Delta_{\eta}^{(a)}(\mathbf{p})=-\left[\Delta_{-\eta}^{(a)}(-\mathbf{p})\right]^{T}$ due to the anti-commutation relations of fermionic creation and annihilation operators. In particular, $\Delta_{K}$ and $\Delta_{-K}$ are not independent gap functions.

The one-loop vertex flow correction is then

$$
\left[\delta \Delta_{\eta}(\mathbf{p})\right]_{\alpha \beta}=\Pi_{\eta^{\prime}} \int[V(\mathbf{k} ; \mathbf{p})]_{\eta ; \eta^{\prime}}^{\alpha \beta ; \alpha^{\prime} \beta^{\prime}}\left[\Delta_{\eta^{\prime}}(\mathbf{k})\right]_{\alpha^{\prime} \beta^{\prime}} \frac{d \theta_{\eta^{\prime}, \mathbf{k}}}{2 \pi}
$$

with a sum over repeated indices implied. This reduces to a system of $2 \times 2$ equations for the $D_{\eta}^{(a)}$ coefficients:

$$
\frac{d}{d s}\left(\begin{array}{c}
D_{\Gamma}^{(a)} \\
D_{K}^{(a)}
\end{array}\right)=-\left(\begin{array}{cc}
\tilde{g}_{1}^{(a)} & 2 \tilde{g}_{4}^{(a)} \sqrt{\frac{N_{K}}{N_{\Gamma}}} \\
\tilde{g}_{4}^{(a)} \sqrt{\frac{N_{\Gamma}}{N_{K}}} & 2 \tilde{g}_{23}^{(a)}
\end{array}\right)\left(\begin{array}{c}
D_{\Gamma}^{(a)} \\
D_{K}^{(a)}
\end{array}\right)
$$

The eigenvalues of the matrix correspond precisely to the decoupled RG effective couplings $\gamma^{(a \pm)}$; thus, $\gamma^{(a+)}>0$ implies a SC transition in the corresponding $a$ channel.

Even when all $g_{j}$ interactions are purely repulsive, this leads to two possible SC instabilities, provided that one of the inter-pocket interactions, $g_{3}$ or $g_{4}$, overcomes the intra-pocket repulsion promoted by $g_{1}$ and $g_{2}$ - see Eqs 24 25). When $g_{4}$ is dominant, the resulting SC state is a singlet $s$-wave, with isotropic gaps $\Delta_{\eta}^{(s)}$. With repulsive interactions, sgn $\left[D_{\Gamma}^{(s)}\right]=-\operatorname{sgn}\left[D_{K}^{(s)}\right]$, the so-called extended $s$-wave or $s^{ \pm}$-wave state, previously proposed to be realized e.g. in iron pnictides [37] and strontium titanate 38 .

In contrast, when $g_{3}$ is the dominant interaction, the SC instability is towards a triplet $f$-wave state, characterized by $\left(\Delta_{\Gamma}(\mathbf{p})\right)_{\alpha \beta} \propto \cos 3 \theta_{\Gamma, \mathbf{p}}\left[(\hat{\mathbf{d}} \cdot \boldsymbol{\sigma}) i \sigma^{y}\right]_{\alpha \beta}$ and $\left(\Delta_{ \pm K}(\mathbf{p})\right)_{\alpha \beta} \propto\left[(\hat{\mathbf{d}} \cdot \boldsymbol{\sigma}) i \sigma^{y}\right]_{\alpha \beta}$. Because spin is conserved in the absence of SOC and magnetic field, the $\hat{\mathbf{d}}$-vector can point in any direction. Unlike typical triplet gaps, here $\Delta_{K}(\mathbf{p})$ is momentum independent on the $K$-pocket, since for triplet states we have $\Delta_{-K}(\mathbf{p})=-\Delta_{K}(\mathbf{p})$. In order for $\Delta_{\Gamma}(\mathbf{p})$ to be nonzero, we include the sub-leading momentum-directiondependent interactions 12 , which do not contribute significantly to the pairing instability. While here our focus is on SC due to purely electronic interactions, the $\mathrm{SC}$ states obtained above are not necessarily inconsistent with electron-phonon interactions, which are expected to promote intra-pocket attraction, thus reducing the amplitude - or even changing the sign - of the $g_{1}, g_{2}$ terms.

\section{Density Wave Instabilities}

Next, we show that the interactions $g_{1}, \ldots g_{4}$ do not lead to density-wave particle-hole instabilities in the spin and charge channels within logarithmic accuracy. This means that any instability in these channels requires a threshold value for these interactions, and is thus unlikely to be driven by the low-energy fermions. The vertices associated with the particle-hole instabilities are

$$
\Delta_{\eta, \eta^{\prime}}^{(\mu D W)} d_{\eta \alpha}^{\dagger} \sigma_{\alpha \beta}^{\mu} d_{\eta^{\prime} \beta}
$$

with $\mu=0$ corresponding to $\mathrm{CDW}$ and $\mu=x, y, z$ to SDW order parameters (here we are ignoring the small momentum dependence). Because the pockets at $K$ and $\Gamma$ are both hole pockets, the spin and charge densitywave channels completely decouple from the SC channels. Explicitly, while the particle-particle bubble

$$
\Pi_{\eta}=\int \frac{\tanh \frac{\epsilon_{\eta}}{2 T}}{2 \epsilon_{\eta}} \frac{d^{2} Q}{(2 \pi)^{3}}
$$

has a logarithmic divergence when integrated over all momenta, the particle-hole bubble does not:

$$
\begin{gathered}
\chi_{\eta}=T \sum_{\omega} \int G_{\eta}^{(0)}(i \omega, \mathbf{Q}) G_{-\eta}^{(0)}(i \omega,-\mathbf{Q}) \frac{d^{2} Q}{(2 \pi)^{3}} \\
=-\int \frac{\operatorname{sech} \frac{\epsilon_{\eta}}{2 T}}{4 T} \frac{d^{2} Q}{(2 \pi)^{2}}
\end{gathered}
$$

Consequently, any particle-hole channel is subleading to the SC channel within weak-coupling. For example,

$$
\delta \Delta_{\Gamma, \Gamma}^{(C D W)}=2 \chi_{\Gamma} g_{1} \Delta_{\Gamma, \Gamma}^{(C D W)}+\chi_{K} \frac{g_{6}+g_{7}}{2} \Delta_{K, K}^{(C D W)}
$$

There are additional CDW and SDW vertices, but all the equations have $\chi_{\eta}$ in them and thus the flows are all exponentially suppressed by a factor of $\operatorname{sech} \frac{\Lambda}{2 T} \approx 2 e^{-\frac{\Lambda}{2 T}}$ as a result. This agrees with the analysis of Ref. 36, which only found leading particle-hole instabilities because the $\Gamma$ pocket was electron-like and nested with the $K$ pocket. Note that an incommensurate CDW has in fact been observed in monolayer $\mathrm{NbSe}_{2}$ 32, as well as in bulk. While its origin remains controversial, the evidence supports a scenario in which the CDW is not a consequence of Fermi surface nesting, consistent with our calculation 31, 3941.

\section{SUPERCONDUCTIVITY IN THE PRESENCE OF SOC AND MAGNETIC FIELD}

The parquet RG treatment of the previous section shows that superconductivity is the only instability generated by the interactions in Eq. (9) within weakcoupling. If the superconducting transition temperature was larger than the Fermi energy, then the superconducting problem would have been essentially solved. However, since $T_{c} \ll E_{F}$ in $\mathrm{NbSe}_{2}$, one needs to proceed to energy scales below the Fermi energy, where Ising and Rashba $\mathrm{SOC}$ and the magnetic field become relevant. Since superconductivity is the leading instability and decouples from other channels below the Fermi energy, it is sufficient to consider a simpler mean-field approach to include the additional terms in the Hamiltonian that were neglected in analysis above. 


\section{A. Mean-Field Gap Equation in the Presence of SOC and Magnetic Field}

In order to determine the appropriate BCS gap equation, we first project the interactions onto the spin-split Fermi surfaces, by using the transformation (5) between eigenstates of spin and eigenstates of the non-interacting Hamiltonian and restricting the gap functions to involve pairs on the same Fermi surface only. Throughout the phase diagrams shown in Fig. 1, the Fermi surfaces are qualitatively similar to those shown in Fig. 2, with the important caveat that in the presence of both Rashba SOC and magnetic field inversion symmetry is broken, as we discuss in more detail below. Our analysis assumes that the minimum splitting between Fermi surfaces (with an associated energy scale on the order of the magnetic field $b$ and/or Rashba SOC $\alpha_{R} p_{F}$ ) is large compared to the superconducting pairing strength, whose energy scale is on the order of the superconducting gap function. In particular, close to the phase transition at which the gap function vanishes this is valid almost everywhere in our phase diagram. Note, however, that for $\alpha_{R}=b=0$, or for $\alpha_{R}=b$ with $\mathbf{B}$ along a $\Gamma-K$ line, the inner and outer Fermi surfaces at the $\Gamma$ pocket touch along the $\Gamma-M$ lines, and our approach is insufficient to resolve the gap in the immediate vicinity of these loci even very close to $T_{c}$.

After the projection, the interactions generally acquire a dependence on the momentum direction. Explicitly, this gives:

$$
\begin{aligned}
H_{\text {Int }}= & \tilde{V}_{\Gamma, \Gamma}^{\tau, \tau^{\prime}} c_{\Gamma \tau}^{\dagger} c_{\Gamma \tau}^{\dagger} c_{\Gamma \tau^{\prime}} c_{\Gamma \tau^{\prime}}+ \\
& \tilde{V}_{ \pm K, \pm K}^{\tau, \tau^{\prime}} c_{ \pm K \tau}^{\dagger} c_{\mp K \tau}^{\dagger} c_{ \pm K \tau^{\prime}} c_{\mp K \tau^{\prime}}+ \\
& \tilde{V}_{ \pm K, \mp K}^{\tau, \tau^{\prime}} c_{ \pm K \tau}^{\dagger} c_{\mp K \tau}^{\dagger} c_{\mp K \tau^{\prime}} c_{ \pm K \tau^{\prime}}+ \\
& \tilde{V}_{\Gamma \pm K}^{\tau, \tau^{\prime}} c_{ \pm K \tau}^{\dagger} c_{\mp K \tau}^{\dagger} c_{\Gamma \tau^{\prime}} c_{\Gamma \tau^{\prime}}
\end{aligned}
$$

with

$$
\tilde{V}_{\eta, \eta^{\prime}}^{\tau, \tau^{\prime}}(\mathbf{p}, \mathbf{k})=\frac{1}{2} \sum_{\mu=0, x, y, z} g_{\eta, \eta^{\prime}}^{(\mu)} Q_{\eta \tau}^{(\mu)}(\mathbf{p}) Q_{\eta^{\prime} \tau^{\prime}}^{(\mu) *}(\mathbf{k})
$$

$\left(\eta, \eta^{\prime}=\Gamma, \pm K\right)$. Here terms on the right-hand side with $\mu=0$ are projections of singlet interactions in (10), while those with $\mu=j \equiv x, y, z$ are projections of the three triplet interactions respectively, into the relevant Fermi surface. Since spin is not conserved in the presence of SOC and/or magnetic field, these three spin polarizations are no longer equivalent. Explicitly,

$$
\begin{aligned}
Q_{\eta \tau}^{(0)}(\mathbf{p}) & =\sum_{\alpha \beta}\left(i \sigma^{y}\right)_{\alpha \beta} U_{\eta \tau}^{\alpha}(\mathbf{p}) U_{-\eta \tau}^{\beta}(-\mathbf{p}) \\
Q_{ \pm K \tau}^{(j)}(\mathbf{p}) & = \pm \sum_{\alpha \beta}\left(i \sigma^{j} i \sigma^{y}\right)_{\alpha \beta} U_{K \tau}^{\alpha}(\mathbf{p}) U_{-K \tau}^{\beta}(-\mathbf{p}) \\
Q_{\Gamma \tau}^{(j)}(\mathbf{p}) & =\sqrt{2} \cos \left(3 \theta_{\mathbf{p}}\right) \sum_{\alpha \beta}\left(i \sigma^{j} i \sigma^{y}\right)_{\alpha \beta} U_{\Gamma \tau}^{\alpha}(\mathbf{p}) U_{\Gamma \tau}^{\beta}(-\mathbf{p}) .
\end{aligned}
$$

with $N_{\eta \tau}$ the density of states at the inner or outer Fermi surface at the $\eta$ pocket and $\Lambda$ is the cut-off energy. Although the Fermi surface is only inversion symmetric when $\alpha_{R}=0$ or $b=0$, the expression 40 is approximately correct when either is sufficiently small; this issue will be discussed in more detail in Sec. IV C
There is a phase ambiguity in the definitions of $Q_{\eta \tau}^{(\mu)}(\mathbf{p})$; the additional factors of $i$ in the last two expressions are chosen to simplify the gap equation below. Here $g_{\eta, \eta^{\prime}}^{(\mu)}$ are constants independent of $\mathbf{p}$ and $\mathbf{k}$ (with $j=x, y, z$ ):

$$
\begin{gathered}
g_{\Gamma, \Gamma}^{(0)}=g_{1} \quad g_{\Gamma, \Gamma}^{(j)}=g_{1}^{t} \\
g_{\Gamma, \pm K}^{(0)}=g_{ \pm K, \Gamma}^{(0)}=g_{4} \\
g_{\Gamma, \pm K}^{(j)}=g_{ \pm K, \Gamma}^{(j)}=g_{4}^{t} \\
g_{ \pm K, \pm K}^{(0)}=g_{ \pm K, \mp K}^{(0)}=\frac{g_{2}+g_{3}}{2} \\
g_{ \pm K, \pm K}^{(j)}=g_{ \pm K, \mp K}^{(j)}=\frac{g_{2}-g_{3}}{2}
\end{gathered}
$$

where $g_{1}, \ldots g_{4}$ are the values of the couplings at the end of the RG procedure described above.

For uniform SC (i.e. with zero center-of-mass momentum), the paired electrons are either both from inner pockets or both from outer pockets, with corresponding gap functions

$$
\begin{aligned}
\Delta_{\Gamma \tau}(\mathbf{p}) & \propto\left\langle c_{\Gamma, \mathbf{p} \tau} c_{\Gamma,-\mathbf{p} \tau}\right\rangle \\
\Delta_{ \pm K \tau}(\mathbf{p}) & \propto\left\langle c_{ \pm K, \mathbf{p} \tau} c_{\mp K,-\mathbf{p} \tau}\right\rangle
\end{aligned}
$$

where the momentum $\mathbf{p}$ is measured with respect to the center of the relevant Fermi pocket. The gaps are diagonal in the index $\tau$, as we assume there is no pairing between inner and outer Fermi surfaces. Note that particlehole symmetry imposes $\Delta_{-K \tau}(\mathbf{p})=-\Delta_{K \tau}(-\mathbf{p})$, so these are not two separate order parameters. The new self-consistent gap equation, analogous to Eq. (27), is (see Appendix A):

$$
\Delta_{\eta \tau}(\mathbf{p})=\sum_{\eta^{\prime}, \tau^{\prime}} \oint \Pi_{\eta^{\prime} \tau^{\prime}}\left(\theta_{\eta^{\prime}, \mathbf{k}}\right) \tilde{V}_{\eta, \eta^{\prime}}^{\tau, \tau^{\prime}}(\mathbf{p} ; \mathbf{k}) \Delta_{\eta^{\prime} \tau^{\prime}}(\mathbf{k}) \frac{d \theta_{\eta^{\prime}, \mathbf{k}}}{2 \pi}
$$

After integrating over momenta, the (angle resolved) particle-particle pairing susceptibility $\Pi_{\eta \tau}\left(\theta_{\eta, \mathbf{p}}\right)$ becomes:

$$
\Pi_{\eta \tau}\left(\theta_{\eta, \mathbf{p}}\right)=-\sum_{p} \frac{\tanh \left(\frac{\beta \xi_{\eta \tau}(\mathbf{p})}{2}\right)+\tanh \left(\frac{\beta \xi_{-\eta \tau}(-\mathbf{p})}{2}\right)}{\xi_{\eta \tau}(\mathbf{p})+\xi_{-\eta \tau}(-\mathbf{p})}
$$

where $\theta_{\eta, \mathbf{p}}$ is the angle along the $\tau$ Fermi surface relative to the center of the pocket $\eta$ (below we simply use $\theta$ when this is clear from context). Assuming that the Fermi surface is inversion symmetric, we find

$$
\Pi_{\eta \tau}\left(\theta_{\eta, \mathbf{p}}\right)=-N_{\eta \tau} \ln \frac{1.13 \Lambda}{T_{c}}
$$


Plugging Eq. 34 into Eq. (38), we find

$$
\Delta_{\eta \tau}(\mathbf{k})=\sum_{\mu} D_{\eta \tau}^{(\mu)} Q_{\eta \tau}^{(\mu)}(\mathbf{p})
$$

where $\mathbf{D}_{\eta \tau}=\left(D_{\eta \tau}^{(0)}, D_{\eta \tau}^{(x)}, D_{\eta \tau}^{(y)}, D_{\eta \tau}^{(z)}\right)$ are momentum independent gap coefficients. The structure of Eq. (38) implies that we can take $D_{\eta \tau}^{(\mu)}=D_{\eta-\tau}^{(\mu)} \equiv D_{\eta}^{(\mu)}$, and we thus drop the $\tau$ index on $D$ hereafter. Moreover, particlehole symmetry enforces $D_{K}^{(\mu)}=D_{-K}^{(\mu)}$, consistent with the fact that $\Delta_{-K \tau}(\mathbf{p})=-\Delta_{K \tau}(-\mathbf{p})$. Plugging the form (41) back into the gap equation (38) yields the reduced gap equations

$$
\begin{aligned}
& D_{\Gamma}^{(0)}=\sum_{\mu}\left(g_{1} f_{(\mu)}^{(0) \Gamma} D_{\Gamma}^{(\mu)}+2 g_{4} f_{(\mu)}^{(0) K} D_{K}^{(\mu)}\right) \\
& D_{K}^{(0)}=\sum_{\mu}\left(g_{4} f_{(\mu)}^{(0) \Gamma} D_{\Gamma}^{(\mu)}+\left(g_{2}+g_{3}\right) f_{(\mu)}^{(0) K} D_{K}^{(\mu)}\right) \\
& D_{\Gamma}^{(j)}=\sum_{\mu}\left(g_{1}^{t} f_{(\mu)}^{(j) \Gamma} D_{\Gamma}^{(\mu)}+2 g_{4}^{t} f_{(\mu)}^{(j) K} D_{K}^{(\mu)}\right) \\
& D_{K}^{(j)}=\sum_{\mu}\left(g_{4}^{t} f_{(\mu)}^{(j) \Gamma} D_{\Gamma}^{(\mu)}+\left(g_{2}-g_{3}\right) f_{(\mu)}^{(j) K} D_{K}^{(\mu)}\right)
\end{aligned}
$$

where $j=x, y, z$ and the form factors $f_{(\mu)}^{\left(\mu^{\prime}\right) \eta}$ are given by

$$
f_{(\mu)}^{\left(\mu^{\prime}\right) \eta}=\frac{1}{2} \oint \sum_{\tau} \Pi_{\eta \tau} Q_{\eta \tau}^{(\mu) *} Q_{\eta \tau}^{\left(\mu^{\prime}\right)} \frac{d \theta_{\eta, \mathbf{k}}}{2 \pi}
$$

In the presence of SOC and magnetic field none of the channels $(\mu=0, x, y, z)$ decouple in general. Eqs. (42) thus can be viewed as an $8 \times 8$ matrix equation, leading to 8 possible superconducting solutions, of which we choose the one with the highest $T_{c}$. The choice of phases in Eq. 35 was made to make the form factors $f_{(\mu)}^{\left(\mu^{\prime}\right) \eta}$ real when the densities of state on inner and outer Fermi surfaces are equal in which case the coefficients $D_{\eta}^{(\mu)}$ can also be taken to be real.

\section{B. Phase Diagrams}

To study the possible superconducting phases, it is useful to define singlet and triplet instability regimes by considering the limit of no SOC and magnetic field. We define a dominant singlet (dominant triplet) instability to occur when the largest eigenvalue of the gap equation is for the spin singlet (spin triplet) gap with SOC and magnetic field set to 0 . The transition temperature for each channel (when the corresponding eigenvalue of the gap equation equals 1 ) is determined by the couplings $g_{1}, \ldots g_{4}$ via

$$
T_{c}^{(a)}=1.13 \Lambda e^{-1 / \gamma^{(a+)}},
$$

where $\Lambda$ is the upper energy cutoff, and $\gamma^{(a+)}$ are identical to the couplings obtained in the RG analysis in Eq. (23) ( $a=s, t$ for singlet and triplet respectively). Fig. 1 shows the phase diagrams corresponding to singlet (a) and triplet (b) instability regimes as a function of Rashba
SOC and in-plane magnetic field. We emphasize that the resulting $\mathrm{SC}$ states themselves are always a mixture of singlet and triplet Cooper pairs.

As seen in Eqs. 24 and 25, with equal DOS in all bands, for repulsive interactions the singlet instability dominates for large $g_{4}$, while the triplet instability dominates for large $g_{3}$. For concreteness, in this section we take

$$
g_{2}=1.2 g_{1}, \quad g_{4}=2 g_{1}, \quad g_{1}^{t}=0.2 g_{1}, \quad g_{4}^{t}=0.1 g_{1}
$$

with $g_{3}=1.05 g_{1}\left(4.2 g_{1}\right)$ to produce a singlet (triplet) instability.

To describe the phase diagrams in Fig. 1, it is useful to classify the solutions to the gap equations (41) by the irreducible representations (irreps) of the relevant point group. In the absence of Rashba SOC and magnetic field, the point group of $1 \mathrm{H}-\mathrm{NbSe}_{2}$ is $D_{3 h}$ 42. We find that the $\mu=0, z$ terms on the right-hand side of Eq. (41) both belong to the $A_{1}^{\prime}$ irrep of $D_{3 h}$, indicating that the singlet and $z$-polarized spin-triplet gaps are mixed in the presence of Ising SOC. In our model, this mixing is proportional to the difference between the densities of states $N_{\eta \tau}$ on the inner $(\tau=-1)$ and outer $(\tau=1)$ Fermi surfaces. Since these densities of states are not expected to differ significantly in two dimensions, this mixing is weak in our model. The remaining components $\mu=x, y$ of the triplet gap transform as the two dimensional $E^{\prime \prime}$ irrep of $D_{3 h}$. We find that, in the absence of both Rashba SOC and magnetic field, the highest $T_{c}$ corresponds to the $A_{1}^{\prime}$ irrep in our model.

Rashba SOC transforms as the $A_{2}^{\prime \prime}$ irrep of $D_{3 h}$. This lowers the point group to $C_{3 v}$, but does not mix the $A_{1}^{\prime}$ and $E^{\prime \prime}$ gaps. In contrast, the in-plane magnetic field $\mathbf{b}$ transforms according to the $E^{\prime \prime}$ irrep of $D_{3 h}$. As a result, it mixes the $E^{\prime \prime}$ gap with the $A_{1}^{\prime}$ one 21. Thus, in the presence of an in-plane magnetic field, all $\mu$ terms in Eq. 41) are mixed.

The electronic spectrum in the superconducting phase is obtained by diagonalizing the Bogolyubov-de Gennes (BdG) Hamiltonian:

$$
H=\frac{1}{2} \sum_{\mathbf{p} \eta \tau} \Psi_{\mathbf{p} \eta \tau}^{\dagger} \mathcal{H}_{\eta \tau}(\mathbf{p}) \Psi_{\mathbf{p} \eta \tau}
$$

where $\Psi_{\eta \tau}(\mathbf{p})=\left(c_{\eta, \mathbf{p} \tau}, c_{-\eta,-\mathbf{p} \tau}^{\dagger}\right)^{T}$ and

$$
\mathcal{H}_{\eta \tau}(\mathbf{p})=\left(\begin{array}{cc}
\xi_{\eta \tau}(\mathbf{p}) & \Delta_{\eta \tau}(\mathbf{p}) \\
\Delta_{\eta \tau}^{*}(\mathbf{p}) & -\xi_{-\eta \tau}(-\mathbf{p})
\end{array}\right)
$$

with $\xi_{\eta \tau}(\mathbf{p})$ given in Eq. (4). Note that when time reversal symmetry (TRS) is broken, $\xi_{-\eta \tau}(-\mathbf{p}) \neq$ $\xi_{\eta \tau}(\mathbf{p})$ in general. The $\mathrm{BdG}$ spectra are given by $E_{\eta \tau}(\mathbf{p}),-E_{-\eta \tau}(-\mathbf{p})$, with:

$$
E_{\eta \tau}(\mathbf{p})=\xi_{A \eta \tau}(\mathbf{p})+\sqrt{\xi_{S \eta \tau}(\mathbf{p})^{2}+\left|\Delta_{\eta \tau}(\mathbf{p})\right|^{2}}
$$




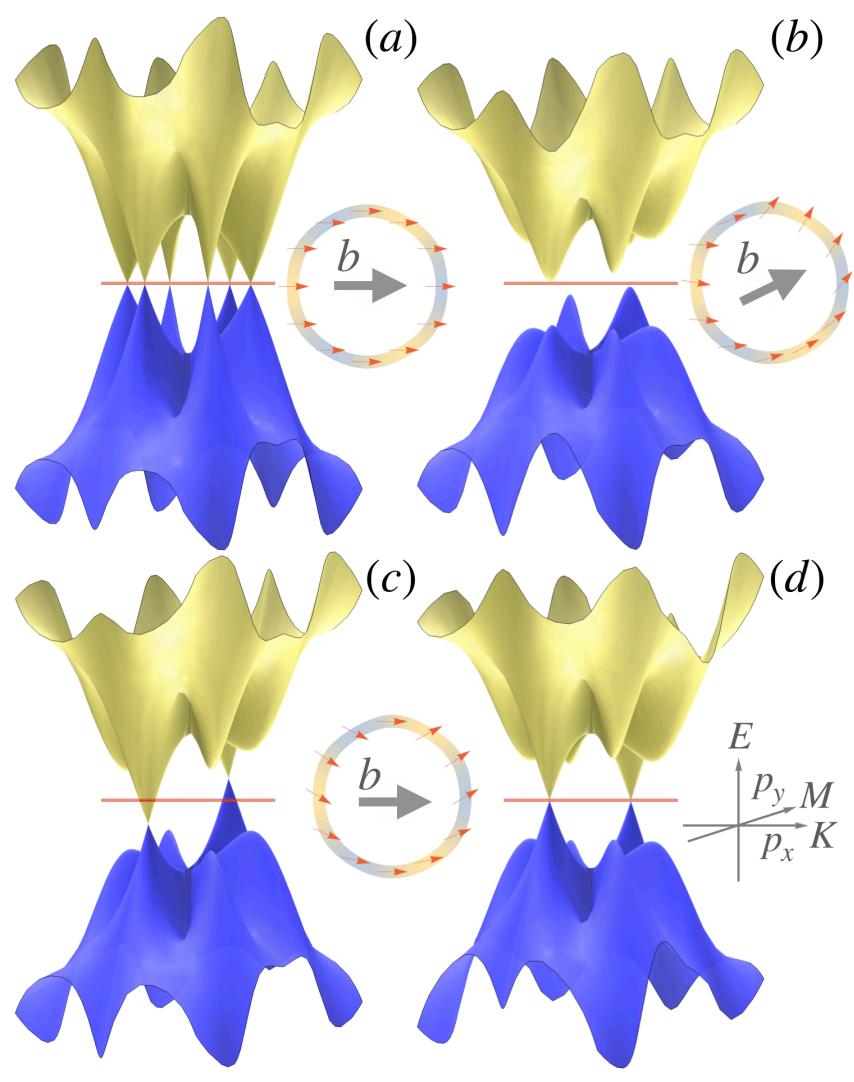

FIG. 3. Superconducting excitation spectrum, Eq. 48, for the inner Fermi surface at $\Gamma$ in the presence of an in-plane magnetic field and without (panel a) or with (panels b, c, d) Rashba SOC. In panel b (panels c and d), B is aligned along $\vartheta=\pi / 7(\vartheta=0)$ from the $\Gamma-K$ lines; panel a is the same for any field direction. In panel c (panel d), the Cooper pair has zero (non-zero) center-of-mass momentum. Insets show the resulting spin textures along the normal-state Fermi surfaces for the corresponding field directions, with colors as in Fig. 2 and arrows indicating in-plane spin components. We used the normal state dispersion from Eq. (2) with the same parameter values given in Eq. (8), and took $b=5 \beta_{I}$, as well as $\alpha_{R}=2 \beta_{I}$ in panels b-d. We set $D_{\Gamma,-1}^{(0)}=20 \beta_{I}$ and $D_{\Gamma,-1}^{(z)}=20 \beta_{I}$ in Eq. 41, and $p_{\text {shift }}=-0.3 p_{F}$ in panel d.

where

$$
\begin{aligned}
\xi_{S \eta \tau}(\mathbf{p}) & =\frac{\xi_{\eta \tau}(\mathbf{p})+\xi_{-\eta \tau}(-\mathbf{p})}{2} \\
\xi_{A \eta \tau}(\mathbf{p}) & =\frac{\xi_{\eta \tau}(\mathbf{p})-\xi_{-\eta \tau}(-\mathbf{p})}{2}
\end{aligned}
$$

Clearly, nodes only occur if both $\xi_{S \eta \tau}$ and $\Delta_{\eta \tau}$ vanish simultaneously. Note that, in our case, the Fermi surface is symmetric under momentum inversion, $\mathbf{p} \rightarrow-\mathbf{p}$, only when either $\alpha_{R}$ or $b$ vanish. In this case, $\xi_{A \eta \tau}=0$ and $\xi_{S \eta \tau}=\xi_{\eta \tau}$.

We are now in position to describe the SC phase diagrams of Fig. 1. obtained for a fixed value of the Ising SOC and varying the magnitude of the magnetic field $b$ and Rashba SOC $\alpha_{R}$. In all cases, the gap at the $\pm K$ pockets is nearly isotropic, so we focus on the $\Gamma$ pocket.
We first analyze the phase diagram of Fig. 11(a), where the dominant $g_{4}$ interaction gives the singlet extended $s$-wave state in the limit of vanishing SOC and magnetic field. Along the $b=0$ axis, the main effect of increasing the Rashba SOC $\alpha_{R}$ is to change the anisotropy of $\Delta_{\Gamma \tau}(\mathbf{p})$, due to the small admixture with the $\mu=z$ nodal triplet gap. Importantly, no phase transition happens along this axis, as the dominant instability is always in the $A_{1}^{\prime}$ irrep of the original $D_{3 h}$ point group. In contrast, along the $\alpha_{R}=0$ axis, a phase transition takes place to a nodal topological SC state for $b=b_{P}$, where $b_{P} \approx \Delta_{\Gamma 1}$ corresponds roughly to the Pauli-limiting field [43, 44. Because our assumption of well-separated Fermi surfaces is not valid for fields smaller than $b_{P}$, our analysis is not sufficient to determine the phase boundary quantitatively, but we show it qualitatively in Fig. 1(a). This phase transition, and the topological character of the resulting nodal SC state, were previously predicted in Ref. 17. and can be understood as a consequence of the vanishing of the Ising SOC along the six $\Gamma-M$ directions, where the SC gap vanishes and 12 nodes ( 6 for each $\Gamma$ Fermi surface) appear due to spins aligning with the magnetic field. This gap structure is shown in the BdG spectrum of the inner $\Gamma$ Fermi surface, displayed in Fig. 3(a) together with the spin texture of the normal-state Fermi surface.

Turning on the Rashba SOC introduces a second spinorbit energy scale that does not vanish along the $\Gamma-M$ directions. As a result, generally even an infinitesimal Rashba SOC lifts the nodes and destroys the topological character of this state, as shown by the fully gapped BdG spectrum in Fig. 3(b). The only exception is when $\mathbf{b}$ is aligned along one of the $\Gamma-K$ directions: in this case, as we discuss in detail in Sec. V, the system has a mirror symmetry. For $\alpha_{R} p_{F}<b$, this symmetry forces spins along the $\Gamma-M$ line perpendicular to $\mathbf{b}$ to align (antialign) with the magnetic field on the inner (outer) $\Gamma$ pocket, as shown in the inset of Fig. 3(c). As a consequence, the gap vanishes along the line perpendicular to $\mathbf{b}$, as displayed by the BdG spectrum of Fig. 3(c). Therefore, two pairs of nodes originally present on this line are protected, whereas the remaining eight nodes are gapped, resulting in a crystalline gapless topological SC state. Because the Fermi surfaces are no longer symmetric under momentum inversion (i.e. $\xi_{A \eta \tau} \neq 0$ ), these protected nodes are generally shifted away from the Fermi level, resulting in the Bogolyubov Fermi surfaces shown in Fig. 3(c). As we discuss in the next section, however, the nodes can move back to the Fermi level if the Cooper pair acquires a finite center-of-mass momentum, which is expected to happen for large enough $b$ and $\alpha_{R}$ (Fig. 3(d)). For $\alpha_{R} p_{F}>b$, however, the pair of nodes on the inner and outer Fermi surfaces merge and the superconducting state becomes fully gapped. While we could not precisely locate this phase boundary, it is expected to interpolate between $b=\alpha_{R} p_{F}$ for large values of $\alpha_{R}$ to $b=b_{P}$ for $\alpha_{R}=0$, as shown by the dashed line in Fig. 1 (a). The evolution of the gap at the outer $\Gamma$ Fermi 
surface across this transition is shown in Figs. 4(a) and (b). In panel (a), the magnetic field is applied along a direction that does not coincide with the $\Gamma-K$ direction. As a result, the nodal superconducting state only exists for $\alpha_{R}=0$ (red curve). In contrast, when the magnetic field is applied along the $\Gamma-K$ direction (panel (b)), two nodes persist even when $\alpha_{R} \neq 0$ (dark orange curve).

We now turn to the case of dominant triplet instability shown in Fig. 1(b), obtained for a dominant $g_{3}$ interaction. As in the singlet regime, we observe a nodal topological superconductor for $\alpha_{R}=0$. In the triplet regime, however, the superconducting gap on the $\Gamma$ pocket is nodal along the entire $\alpha_{R}=0$ line (except for very small magnetic fields, where the small difference in density of states on the inner and outer Fermi surfaces can open a gap); hence the nodal topological SC state occurs for all values of $b$, as there is no Pauli limit in this case [43, 44. Similarly, the transition into the crystalline nodal topological phase happens close to the $\alpha_{R} p_{F}=b$ line.

Along the $b=0$ line, the nodes on the $\Gamma$ pocket in the $\mu=z$ triplet state are lifted due to the admixture with the sub-leading s-wave $\mu=0$ state generated by Ising SOC, resulting in an anisotropic gap. This is shown in Fig. 4(c), which presents the evolution of $\Delta_{\Gamma 1}(\mathbf{p})$ along the $b=0$ axis for increasing $\alpha_{R}$. Note that to generate singlet-triplet mixing, we must include a small difference between the inner and outer DOS in the plots of Fig. 4, the magnitude of this mixing increases with $\alpha_{R}$. Thus, although the superconducting gap on $\Gamma$ in the triplet regime remains strongly modulated as a function of angle for modest $\alpha_{R}$ (see Fig. 4(c)), the superconducting phase in this region is nonetheless fully gapped.

For large values of $\alpha_{R}$ (of the order of the Ising SOC), the dominant instability shifts from being in the $A_{1}$ irrep of $C_{3 v}$ (previously the $A_{1}^{\prime}$ irrep of the original $D_{3 h}$ group) to the 2-dimensional $E$ irrep (previously the $E^{\prime \prime}$ irrep of the original $D_{3 h}$ group). As a result, a new chiral $p \pm i p$ superconducting state emerges in the triplet regime at $b=0$. The chiral phase, shown in Fig. 4(d), occurs because in this 2-dimensional irrep the free energy is minimized by a spontaneous breaking of time reversal symmetry, as we discuss in Appendix B. This is in agreement with the general result in [45, 46. As we show in Appendix B, this results in a gapped chiral topological SC with gapless chiral edge modes resulting in a thermal Hall conductance $\kappa_{x y}= \pm 6\left(\pi^{2} k_{B}^{2} / 3 h\right) T[28$. This topological SC phase survives for sufficiently small in-plane magnetic fields, but our approach is insufficient to quantitatively obtain the phase boundary (see blue dashed line in Fig. 1(b)).

\section{Broken Momentum Inversion Symmetry: Bogolyubov Fermi Surfaces and Finite-Momentum Pairing}

In the presence of both $\alpha_{R}$ and $b$, the Fermi surfaces are no longer inversion-symmetric under $\mathbf{p} \rightarrow-\mathbf{p}$. The key

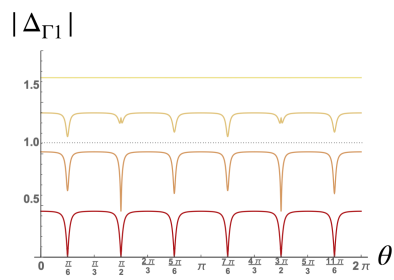

(a)

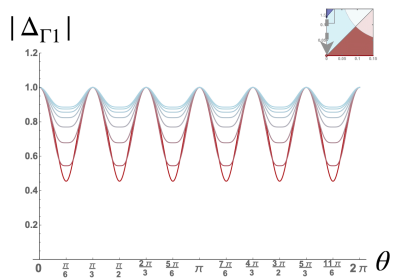

(c)

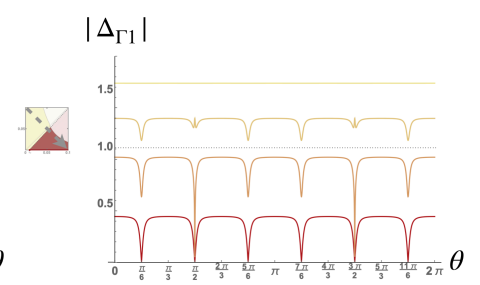

(b)

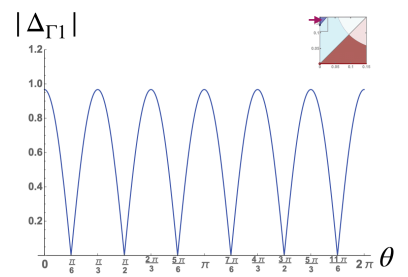

(d)
FIG. 4. Superconducting gap $\Delta_{\Gamma 1}$ in Eq. 41) at the outer $\Gamma$ pocket, as a function of the angle $\theta$ along the Fermi surface with respect to the $\Gamma-K$ direction, in various regions of the phase diagrams of Fig. 1. Panels (a) and (b) correspond to the cuts across the phase diagram of Fig. 11(a) shown in the insets, with a magnetic field away from the $\Gamma-K$ direction by an angle $\vartheta=2 \pi / 25$ (panel (a)) and along the $\Gamma$ - $K$ direction (panel (b)). Panels (c) and (d) correspond to cuts along the $b=0$ axis of the phase diagram of Fig. 1 (b), outside and inside the chiral SC phase, respectively (see insets). Note that the gap amplitudes have been rescaled for clarity, since they are not fixed by the linearized gap equations. We used coupling constants given in Eq. (45) and parameters for the non-interacting Hamiltonian as in Eq. 8. We also took the inner and outer densities of states to differ by ten percent to ensure that the symmetry allowed mixings between the singlet and triplet channels are present in our solutions.

quantity that measures the degree of symmetry breaking is

$$
\xi_{A \eta \tau}(\mathbf{p})=\frac{\xi_{\eta \tau}(\mathbf{p})-\xi_{-\eta \tau}(-\mathbf{p})}{2}
$$

previously defined in Eq. 50 . Here we discuss two important consequences of this inversion symmetry breaking for the crystalline topological SC state. First, within the crystalline topological SC state, breaking inversion symmetry moves the two nodes on the same Fermi surface at $\Gamma$ in opposite directions away from the Fermi level. This follows directly from Eq. (48) as the nodes move by an energy $\xi_{A \Gamma \tau}\left(\mathbf{p}_{\text {node }}\right)$. As we showed in Fig. 3(c), this results in the nodes 'inflating' into Bogolyubov Fermi surfaces $(c f$. [47 50]). These Fermi surfaces are protected by mirror symmetry, due to the topological stability of the band crossings in the BdG spectrum, as we show in the next section.

Second, breaking momentum inversion symmetry cuts off the Cooper logarithm in the particle-particle bubble in Eq. 40. As a result, the pairing interaction must be larger than a certain threshold, proportional to how much inversion symmetry is broken, for a uniform SC state to onset. To show this explicitly, we evaluate the 

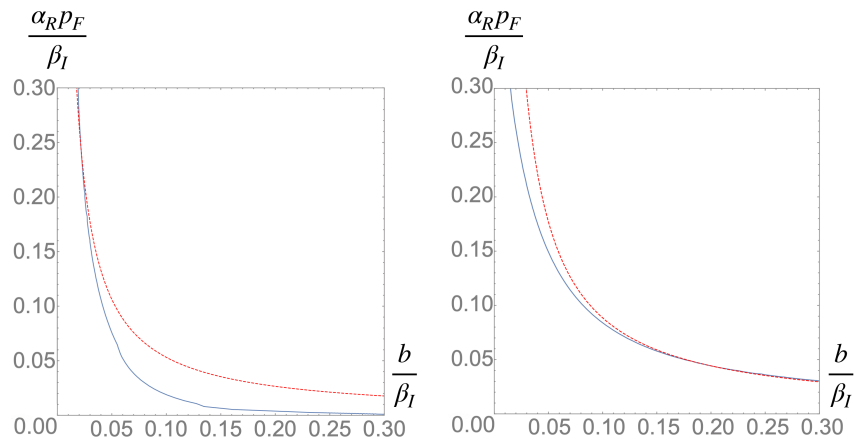

FIG. 5. Critical lines above which uniform SC becomes unstable for the singlet (left) and triplet (right) phase diagrams shown in Fig. 11 Blue line is the numerical solution from the full gap equation, while the red dashed line is given by the approximation $\alpha_{R} p_{F} b=\frac{\beta_{I} T_{c 0}}{1.13}$. In addition to the parameters used in Fig. 4 we took $T_{c}=0.01 \beta_{I}$ and $\Lambda=25 \beta_{I}$, roughly corresponding to the observed values [7, 10, 20].

particle-particle bubble in Eq. (39) in the absence of inversion symmetry. Assuming that $\xi_{A \eta \tau}$ is a function only of the direction $\theta$ around the Fermi surface, in the limit of $\Lambda \gg \xi_{A \eta \tau}$ we find

$\frac{\Pi_{\eta \tau}(\theta)}{N_{\eta \tau}}=-\ln \frac{1.13 \Lambda}{T_{c}}+\operatorname{Re}\left[\psi\left(\frac{1}{2}+\frac{i \xi_{A \eta \tau}(\theta)}{2 \pi T_{c}}\right)-\psi\left(\frac{1}{2}\right)\right]$

where $\psi$ is the digamma function. As a result, at zero temperature

$$
\Pi_{\eta \tau}(\theta)=-N_{\eta \tau} \ln \frac{\Lambda}{\left|\xi_{A \eta \tau}(\theta)\right|}
$$

i.e. the infrared logarithmic divergence originally present is cutoff by $\left|\xi_{A \eta \tau}(\theta)\right|$. This means that there is a critical value of the parameter $\xi_{A \eta \tau}^{c}$ beyond which uniform SC is no longer stable. The resulting critical lines are shown in Fig. 1 and for a larger range in Fig. 5 for both singlet and triplet instabilities. Note that because this is a multi-band superconductor, the critical line has a (weak) dependence on the cutoff $\Lambda$.

The general shape of the critical lines can be understood from a simple approximation, noting that

$$
\xi_{A \eta \tau}(\mathbf{p})=\frac{\tau}{2}\left(\delta_{\eta}(\mathbf{p})-\delta_{-\eta}(-\mathbf{p})\right)
$$

where $\delta_{\eta}$ are functions of $\alpha_{R} p_{F}$ and $b$ as given in 77 . For $\alpha_{R} p_{F}, b \ll \beta_{I}$,

$$
\xi_{A \eta \tau}(\mathbf{p}) \approx \tau \frac{\alpha_{R} p_{F} b}{\beta_{I}} \sin (\theta-\vartheta)
$$

where $\beta_{I}$ is the Ising SOC on the relevant pocket and $\vartheta$ is the direction of the magnetic field. From (53), we can estimate that the characteristic scale of $\xi_{A \eta \tau}^{c}$, properly averaged, is of the order $T_{c 0} / 1.13$, where $T_{c 0}$ is the solution of the gap equation when $\xi_{a \eta \tau}^{c}=0$, see Eq. 40 .
The critical curve is thus roughly given by

$$
\alpha_{R} p_{F} b \sim \frac{\beta_{I} T_{c 0}}{1.13}
$$

As shown in Fig. 5, this approximation reasonably captures the exact result for the critical line.

For inversion symmetry breaking exceeding the critical value, where the uniform SC state is no longer stable, superconductivity with finite center-of-mass momentum $\mathbf{p}_{\text {shift }} \neq 0$, i.e. a so-called FFLO phase [30, 51, 52, is still possible. Note that the momentum shift is not necessarily equal for each Fermi surface, so more generally there are four parameters $\mathbf{p}_{\text {shift }, \eta \tau}$. Depending on whether $\mathbf{p}_{\text {shift }, \eta \tau}=\mathbf{p}_{\text {shift }, \eta-\tau}$ or $\mathbf{p}_{\text {shift }, \eta \tau}=-\mathbf{p}_{\text {shift }, \eta-\tau}$, the FFLO phase is classified as helical and stripe, respectively, and may even compete with the uniform SC phase below the threshold curve in the $\left(\alpha_{R} p_{F}, b\right)$ plane [51. Ultimately, the four parameters $\mathbf{p}_{\text {shift }, \eta \tau}$ must be obtained by minimization of the free energy, which is a computationally involved task beyond the scope of our work. It is interesting to note, however, that by matching $\mathbf{p}_{\text {shift }}$ with the geometric shift of the center of the corresponding Fermi surface, the nodes of the superconducting ground state move back to the Fermi level since the shift compensates for the finite $\xi_{A \eta \tau}$ (see Eqs. (63)(65) below), as shown in Fig. 3(d). Because this configuration maximizes the gap around the Fermi surface, it is expected to maximize the condensation energy. In any case, as we show in the next section, the finite momentum pairing does not affect the topological properties of the SC phase.

\section{CRYSTALLINE GAPLESS TOPOLOGICAL SUPERCONDUCTIVITY}

Having established the existence of a nodal SC phase for large magnetic fields in the phase diagrams of Fig. 1. we now discuss its topological properties. As discussed in Refs. 53 58, two-dimensional gapless topological phases are stable only in the presence of certain symmetries, which guarantee stability of both the bulk nodes and of the corresponding edge modes. When $\alpha_{R}=0$, the SC state has both particle-hole symmetry $\mathcal{C}$ and an anti-unitary time-reversal-like symmetry $\tilde{\mathcal{T}}=i \sigma^{x} \mathcal{K}(\mathcal{K}$ is complex conjugation, and $\sigma^{x}$ acts on the spin index), which is a composition of time-reversal symmetry and a reflection with respect to the $x y$ plane. $\tilde{\mathcal{T}}$ reverses the in-plane momentum and the $z$ component of the spin, satisfying $\tilde{\mathcal{T}}^{2}=1$. This time-reversal-like symmetry places the system into symmetry class BDI [59,61] and protects the 12 nodes of the superconducting gap on the two $\Gamma$ pockets along the $\Gamma-M$ lines, ensuring that the boundary flat bands cannot be gapped [22, 55]. However, a finite Rashba SOC breaks the $\tilde{\mathcal{T}}$ symmetry, putting the model in symmetry class D. For generic in-plane field directions, this results in a fully gapped, topologically 
trivial SC phase with no protected zero-energy boundary states.

The notable exception is when $\mathbf{B}$ is parallel to one of the $\Gamma-K$ directions: in this case, the system has a mirror symmetry associated with reflection about the plane perpendicular to $\mathbf{B}$. For example, when $\mathbf{B}$ is parallel to the $x$ axis, the mirror symmetry corresponds to a reflection with respect to the $y z$ plane perpendicular to $\mathbf{B}$, which also flips the $y$ and $z$ components of spin: $\mathcal{M}_{x}=i \sigma^{x} R_{y z}$, where the reflection $R_{y z}$ corresponds to $(x, y, z) \rightarrow(-x, y, z)$ and, as above, $\sigma^{x}$ acts on the physical spin indices. In general the superconducting gap can be either even or odd under this mirror symmetry. We show below that for $b>\alpha_{R} p_{F}$, where the gap is mirror-odd, the reflection symmetry anti-commutes with particle-hole symmetry. In this case, there is a $\mathbb{Z}$-valued topological invariant that diagnoses the topological superconducting phase [56. This invariant is characterized by non-vanishing quantized winding number along a contour encircling each node - or if the nodes are not at the Fermi energy [60, a contour encircling each Bogolyubov Fermi surface 62. Thus mirror reflection protects the four nodes in the reflection plane provided that $b>\alpha_{R} p_{F}$ (see Fig. 3(c)) [56, 57, 63. When $b=\alpha_{R} p_{F}$, pairs of nodes touch and there is a topological phase transition into a nodeless phase at $b<\alpha_{R} p_{F}$.

We emphasize that the topological nature of this $\alpha_{R} \neq$ $0 \mathrm{SC}$ state is qualitatively different than that of the $\alpha_{R}=0 \mathrm{SC}$ state, as in the former case the symmetry that protects the $\mathrm{SC}$ state is not time-reversal-like, but a mirror symmetry - hence the denomination crystalline gapless topological SC [56, 57.

\section{A. Mirror Symmetry and Topologically Protected Nodes}

To see how the mirror reflection symmetry protects the nodes in the reflection plane, we will follow the approach of Ref. [56], who established that an analysis of the symmetry-allowed mass terms in an effective low energy theory can reveal whether a non-gapped superconductor is topologically non-trivial. $\mathcal{M}_{x}$ acts on the non-interacting Hamiltonian (1) as

$$
\mathcal{M}_{x}^{-1} H(\mathbf{p}) \mathcal{M}_{x}=H(\overline{\mathbf{p}})
$$

where $\overline{\mathbf{p}}=\left(-p_{x}, p_{y}\right)$. Since this reflection also reverses the $y$ and $z$ components of the spin, in the spin basis $\mathcal{M}_{x}$ acts as $i \sigma^{x}$, while in the SOC basis (5) it is momentum dependent, $\mathcal{M}_{x}(\mathbf{p})=U_{\eta \tau}^{\alpha}(\mathbf{p}) i \sigma_{\alpha \beta}^{x} U_{\eta \tau^{\prime}}^{\beta *}(\overline{\mathbf{p}})=i \tau e^{-i \phi(\mathbf{p})} \delta_{\tau \tau^{\prime}}$, where $\phi(\mathbf{p})$ is given in Eq. (7).

The action of mirror symmetry can be extended to the $\mathrm{BdG}$ spinors to give

$$
\begin{aligned}
\tilde{\mathcal{M}}_{x}(\mathbf{p})= & \left(\begin{array}{cc}
\mathcal{M}_{x}(\mathbf{p}) & 0 \\
0 & -\mathcal{M}_{x}^{\dagger}(-\mathbf{p})
\end{array}\right) \\
& =\left(\begin{array}{cc}
e^{i \phi(\mathbf{p})} & 0 \\
0 & -e^{-i \phi(-\mathbf{p})}
\end{array}\right)
\end{aligned}
$$

On the BdG Hamiltonian Eq. (47), the mirror symmetry thus acts according to

$$
\tilde{\mathcal{M}}_{x}^{-1} \mathcal{H}_{\eta \tau}(\mathbf{p}) \tilde{\mathcal{M}}_{x}=\mathcal{H}_{\eta \tau}(\overline{\mathbf{p}})
$$

Here the relative sign between the two non-vanishing components of $\tilde{M}$ is fixed by whether the gap function is even or odd under the mirror symmetry. For $b>\alpha_{R} p_{F}$, where the SC gap that gives the highest $T_{c}$ in Eq. 38 is odd under $p_{x} \rightarrow-p_{x}$, the appropriate choice is minus 64 .

Following the methods of Ref. 56, we will show that in the low-energy theory obtained by linearizing the model near the nodes, there are no symmetry-allowed mass terms; this is equivalent to showing that the topological winding number is non-trivial (which we have also verified in our model by directly computing the Berry connection, though we will not present the calculation here). However, we do find symmetry-allowed terms that shift the nodes away from the Fermi energy, leading to protected Bogolyubov Fermi surfaces.

We begin by linearizing the Hamiltonian in the region $b>\alpha_{R} p_{F}$ around the pair of nodes at $p_{x}=0$. This gives a $4 \times 4$ low-energy effective Hamiltonian, with a new index $L, R$ to keep track of the two nodes. We define $\tau^{\mu}$ to be the Pauli matrices acting on the $L, R$ indices, while $\varsigma^{\mu}$ are Pauli matrices acting on the 2 indices of the BdG spinors (i.e. on the particle-hole indices). In this basis the particle-hole symmetry, which interchanges the two nodes, acts via $\mathcal{C}=\varsigma^{x} \otimes \tau^{x} \mathcal{K}$. Note that Eq. (58) implies that the action of $\mathcal{M}_{x}$ on the mirror plane changes discontinuously at $b=\alpha_{R} p_{F}$ : for $b<\alpha_{R} p_{F}, \mathcal{M}_{x}$ is proportional to the identity matrix times $\operatorname{sgn} p_{y}$, while for $b>\alpha_{R} p_{F}$ it has the form $\tilde{\mathcal{M}}_{x}(\mathbf{p})=\varsigma^{z}$. Since the mirror symmetry acts in the same way near both nodes, in our linearized theory it acts via

$$
\tilde{\mathcal{M}}_{x}=\varsigma^{z} \otimes \tau^{0}
$$

Since the nodes are in the mirror plane, this leading-order approximation is sufficient to determine which terms can open a gap,

We now consider all leading terms of the generic form $h\left(\delta p_{y}, p_{x}\right) \varsigma^{\mu} \otimes \tau^{\nu}$ allowed by symmetry, with $\delta p_{y}=$ $p_{y}-p_{y}^{\text {(node) }}$. Note that we do not wish to allow terms that couple the two nodes, as these break translational symmetry; thus we require $\nu=0$ or $z$. Recall that the symmetries are

$$
\begin{aligned}
\mathcal{C}^{-1} \mathcal{H}\left(\delta p_{y}, p_{x}\right) \mathcal{C} & =-\mathcal{H}\left(-\delta p_{y},-p_{x}\right) \\
\tilde{\mathcal{M}}_{x}^{-1} \mathcal{H}\left(\delta p_{y}, p_{x}\right) \tilde{\mathcal{M}}_{x} & =\mathcal{H}\left(\delta p_{y},-p_{x}\right)
\end{aligned}
$$

Thus $h\left(-\delta p_{y},-p_{x}\right)= \pm h\left(\delta p_{y}, p_{x}\right)$ when $\mathcal{C}^{-1} \varsigma^{\mu} \otimes \tau^{\nu} \mathcal{C}=$ $\mp \varsigma^{\mu} \otimes \tau^{\nu}$. Similarly $h\left(\delta p_{y},-p_{x}\right)= \pm h\left(\delta p_{y}, p_{x}\right)$ when $\tilde{\mathcal{M}}_{x}^{-1} \varsigma^{\mu} \otimes \tau^{\nu} \tilde{\mathcal{M}}_{x}= \pm \varsigma^{\mu} \otimes \tau^{\nu}$. To gap out the nodes we must have $h(0,0) \neq 0$; thus we need plus signs in both cases. Hence $\varsigma^{\mu} \otimes \tau^{\nu}$ anti-commutes with $\varsigma^{x} \otimes \tau^{x} \mathcal{K}$ and commutes with $\tilde{\mathcal{M}}_{x}$. 
This restricts the linearized Hamiltonian for $b>\alpha_{R} p_{F}$ to the form

$$
\mathcal{H}=\delta p_{y} \varsigma^{z} \otimes \tau^{z}+p_{x} \varsigma^{x} \otimes \tau^{0}+m \varsigma^{z} \otimes \tau^{0}+\xi_{A} \varsigma^{0} \otimes \tau^{z}
$$

where $m$ and $\xi_{A}$ are constants (for simplicity, we take the Fermi velocity to be 1 , so all parameters have the same units). The $m$ term, which plays the role of a chemical potential shift at each node, does not lift the nodes; rather it shifts them in opposite directions along the $p_{y}$ axis, from $p_{y}= \pm p_{F}$ to $p_{y}= \pm\left(p_{F}+m\right)$. The $\xi_{A} \varsigma^{0} \otimes \tau^{z}$ term, on the other hand, shifts the nodes in opposite directions in energy by an amount $\xi_{A}$, inflating the nodes into small Bogolyubov Fermi surfaces (see also [47 50]). Comparing with the BdG spectrum Eq. 48), we find that $\xi_{A}$ is precisely the value of $\xi_{A \Gamma \tau}$ given in Eq. (50) when $\xi_{S \Gamma \tau}(\mathbf{p})=0$ in Eq. 49. Since a constant shift in energy cannot change the Berry connection, the winding numbers are unaffected and remain non-trivial [57, 58, as can be verified by direct computation. Note that the Bogolyubov Fermi surfaces are topologically protected only in a fragile sense [65, as they can be removed by mixing with additional bands, similar to what has been observed theoretically in 1D crystalline topological insulators 66, 67.

The term $\xi_{A} \varsigma^{0} \otimes \tau^{z}$ describes a state with broken momentum inversion symmetry, in which the normal state Fermi surface is shifted by a momentum of $\xi_{A}$ relative to the Brillouin zone center. To see this, note that at $p_{x}=0$, the normal state Fermi surface at both nodes is determined by $\delta p_{y}+\xi_{A}=0$. This means that Cooper pairs cannot both lie on the Fermi surface. As noted in Sec. IVC for sufficiently large inversion symmetry breaking, uniform pairing becomes unstable, but an FFLO-type finite-momentum pairing between electrons on the Fermi surface remains a possibility [51. In this case the Nambu spinors have to be redefined as $\left(c_{\mathbf{p}+p_{\text {shift }} \hat{\mathbf{y}}}, c_{-\mathbf{p}+p_{\text {shift }} \hat{\mathbf{y}}}^{\dagger}\right.$, where $2 p_{\text {shift }}$ is the total momentum of the pair. This transforms the BdG Hamiltonian in Eq. 47) into

$$
\mathcal{H}_{\eta \tau}(\mathbf{p})=\left(\begin{array}{cc}
\xi_{\eta \tau}\left(\mathbf{p}+\mathbf{p}_{\text {shift }}\right) & \Delta_{\eta \tau}(\mathbf{p}) \\
\Delta_{\eta \tau}^{*}(\mathbf{p}) & -\xi_{-\eta \tau}\left(-\mathbf{p}+\mathbf{p}_{\text {shift }}\right)
\end{array}\right) .
$$

with the spectrum now given by

$$
E_{\eta \tau}(\mathbf{p})=\xi_{A \eta \tau}\left(\mathbf{p}, \mathbf{p}_{\text {shift }}\right)+\sqrt{\xi_{S \eta \tau}\left(\mathbf{p}, \mathbf{p}_{\text {shift }}\right)^{2}+\left|\Delta_{\eta \tau}(\mathbf{p})\right|^{2}}
$$

with

$$
\begin{aligned}
& \xi_{S \eta \tau}\left(\mathbf{p}, \mathbf{p}_{\text {shift }}\right)=\frac{\xi_{\eta \tau}\left(\mathbf{p}+\mathbf{p}_{\text {shift }}\right)+\xi_{-\eta \tau}\left(-\mathbf{p}+\mathbf{p}_{\text {shift }}\right)}{2} \\
& \xi_{A \eta \tau}\left(\mathbf{p}, \mathbf{p}_{\text {shift }}\right)=\frac{\xi_{\eta \tau}\left(\mathbf{p}+\mathbf{p}_{\text {shift }}\right)-\xi_{-\eta \tau}\left(-\mathbf{p}+\mathbf{p}_{\text {shift }}\right)}{2}
\end{aligned}
$$

Linearizing the transformed BdG Hamiltonian around the nodes (where $\xi_{S \eta \tau}\left(\mathbf{p}, \mathbf{p}_{\text {shift }}\right)=0$ ) yields a new term $-p_{\text {shift }} \varsigma^{0} \otimes \tau^{z}$ in Eq. 62 . Picking $p_{\text {shift }}=\xi_{A}$, this term cancels the energy shift of the nodes, bringing them back to the Fermi level.

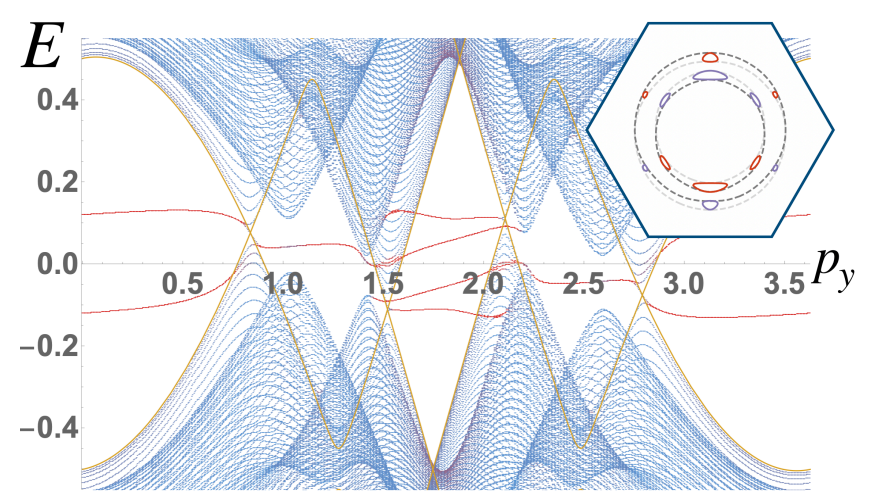

FIG. 6. Excitation spectrum in the topological crystalline SC phase on a $150 \times \infty$ unit cell strip with $\mathbf{B}=B \hat{\mathbf{x}}$. Blue indicates delocalized bulk eigenstates, red indicates eigenstates concentrated near the boundaries, and yellow shows a cut of the bulk BdG spectrum at $p_{x}=0$. The inset illustrates the Bogolyubov Fermi surfaces and the original Fermi surfaces (dashed black lines) and its inverse image under $\mathbf{p} \rightarrow-\mathbf{p}$ (dashed gray lines). For details of the tight-binding model, see Appendix C

\section{B. Boundary modes}

By the bulk-boundary correspondence [56, 57, there are edge bands terminating at the nodes. However, unlike other nodal topological superconductors with Majorana flat band edge modes [17, 53, 54, 68, the edge modes in the crystalline topological phase under consideration are not in general flat and not necessarily at zero energy. The edge bands can be studied following the methods of 69 , 70] we find that for open boundary conditions in $x$ and $p_{y}$ close to the node, the edge mode has energy $\xi_{A}$. When $\xi_{A}$ vanishes at the node (e.g. if a stripe FFLO phase is realized in the bulk) this means that the boundary modes cross zero energy- but they are not flat in general as $\xi_{A}$ does not have to vanish for all $p_{y}$. Similar edge states have been studied in 3D crystalline topological insulators, where they are referred to as drumhead states $71-73$.

An alternative way to understand the edge modes, is to view them as topological boundary modes of the family of $1 \mathrm{D}$ Hamiltonians $\mathcal{H}_{p_{y}}\left(p_{x}\right)=\mathcal{H}\left(p_{x}, p_{y}\right)$ at fixed $p_{y}$, which are in topological class A and respect mirror symmetry. Since mirror symmetry is equivalent to inversion in 1D, these are the same systems as studied in [66, 67. As $p_{y}$ crosses the node, $\mathcal{H}_{p_{y}}\left(p_{x}\right)$ undergoes a topological phase transition from trivial to non-trivial. The 1D topological invariant is the mirror index $M \mathbb{Z}$ defined as follows 66 , 74. Since $\mathcal{H}_{p_{y}}\left(p_{x}\right)$ commutes with $\mathcal{M}_{x}$ at $p_{x}=0$ and $p_{x}=\pi$ (the boundary of the 1D Brillouin zone), at those points it can be decomposed into two blocks on which $\mathcal{M}_{x}= \pm 1$ respectively: $\mathcal{H}_{p_{y}}^{ \pm}(0)$ and $\mathcal{H}_{p_{y}}^{ \pm}(\pi)$. If $n_{+}^{(0)}$ is the number of occupied states of $\mathcal{H}_{p_{y}}^{+}(0)$ and $n_{+}^{(\pi)}$ is that of $\mathcal{H}_{p_{y}}^{+}(\pi)$, the mirror index is simply $N_{M \mathbb{Z}}=\left|n_{+}^{(0)}-n_{+}^{(\pi)}\right|$. There are then $2 N_{M \mathbb{Z}}$ edge states, with each state even under reflection being degenerate with an edge state that 
is odd under reflection. Taken together these edge states form the band of edge modes of the $2 \mathrm{D}$ system. At a node (which is at $\left.p_{x}=0\right), \mathcal{H}_{p_{y}}^{+}(0)$ crosses with a state in $\mathcal{H}_{p_{y}}^{-}(0)$, which changes $n_{+}^{(0)}$ by one and the number of edge states by two. The edge mode thus splits into two bulk modes which cross at the node.

To study the boundary modes of our model in the uniform superconducting state, in Appendix C we describe a tight-binding model that captures the key features of our gapless topological superconducting phase. The results are displayed in Fig. 6, which shows the Bogolyubovde Gennes $(\mathrm{BdG})$ spectrum on a $150 \times \infty$ unit cell strip with open zig-zag edges parallel to the $\hat{y}$ direction, and $\mathbf{B}=B \hat{x}$, in the uniform superconducting state. Each state $\psi_{k}$ is colored according to the inverse participation ratio $\sum_{y}\left|\psi_{k}(y)\right|^{4}$, such that the boundary modes are red, and bulk modes are blue. A cut containing the nodes along $p_{x}=0$ of the bulk BdG spectrum is shown in yellow.

It is worth noting that in actual materials, the existence of both bulk nodes and corresponding boundary states is guaranteed only if the relevant mirror reflection is an exact symmetry. As such, these may be sensitive to orientational defects in the crystal.

\section{CONCLUDING REMARKS}

Our microscopic interacting model for $\mathrm{NbSe}_{2}$ predicts multiple possible exotic superconducting phases in this material, tuned by the Rashba SOC $\alpha_{R}$ and the in-plane magnetic field $B$. Two different primary SC instabilities (i.e. that would take place when Ising SOC is zero) can be driven by purely electronic interactions: a singlet extended $s$-wave and a triplet $f$-wave instability. The phase diagrams for both are qualitatively similar, with a fully gapped superconductor for $\alpha_{R}>b$, and a crystalline gapless topological SC state for large b and small $\alpha_{R}$. Interestingly, the topological properties of the latter phase depend crucially on the $\mathbf{B}$ field being aligned along one of the $\Gamma-K$ directions. In addition, the triplet instability supports a chiral topological SC state for small $b$ and large $\alpha_{R}$.

Although direct experimental detection of these topological SC states may be technically challenging, their indirect experimental manifestations should be accessible. For instance, because the chiral SC state transforms as a two-dimensional irreducible representation of the trigonal space group, it should be strongly affected by strain, with $T_{c}$ splitting into 2 separate transitions under externally applied uniaxial strain [75. As for the crystalline topological SC state, its extreme sensitivity to the field direction is expected to promote strongly anisotropic properties. Specifically, since the nature of the SC state changes as a function of the B direction, one expects pronounced six-fold anisotropies in the upper critical field and in the critical current. Recent experiments have identified sixfold and two-fold anisotropies in the magneto-resistance and in the critical field [76, 77. Whether these results can be attributed to the crystalline nodal topological superconducting state discussed here requires further investigation. Finally, the presence of Bogolyubov Fermi surfaces should also be manifested in several experimental observables that are sensitive to the existence of a finite DOS at zero energy [78.

\section{ACKNOWLEDGMENTS}

We thank T. Birol, A. Chubukov, V. Pribiag, and K. Wang for fruitful discussions. This work was supported primarily by the National Science Foundation (NSF) Materials Research Science and Engineering Center at the University of Minnesota under Award No. DMR1420013, via an iSuperSeed Award (DS, FJB, and RMF). FJB is grateful for the financial support of the Sloan Foundation FG-2015- 65927. JK was supported by the National High Magnetic Field Laboratory through NSF Grant No. DMR- 1157490 and the State of Florida.
[1] Y. Cao, A. Mishchenko, G. L. Yu, E. Khestanova, A. P. Rooney, E. Prestat, A. V. Kretinin, P. Blake, M. B. Shalom, C. Woods, J. Chapman, G. Balakrishnan, I. V. Grigorieva, K. S. Novoselov, B. A. Piot, M. Potemski, K. Watanabe, T. Taniguchi, S. J. Haigh, A. K. Geim, and R. V. Gorbachev, Nano Letters, Nano Letters 15, 4914 (2015)

[2] J. T. Ye, Y. J. Zhang, R. Akashi, M. S. Bahramy, R. Arita, and Y. Iwasa, Science 338, 1193 (2012)

[3] K. Taniguchi, A. Matsumoto, H. Shimotani, and H. Takagi, Applied Physics Letters 101, 042603 (2012)

[4] X. Xi, H. Berger, L. Forró, J. Shan, and K. F. Mak, Phys. Rev. Lett. 117, 106801 (2016).

[5] J. M. Lu, O. Zheliuk, I. Leermakers, N. F. Q. Yuan, U. Zeitler, K. T. Law, and J. T. Ye, Science 350, 1353
(2015)

[6] Y. Saito, Y. Nakamura, M. S. Bahramy, Y. Kohama, J. Ye, Y. Kasahara, Y. Nakagawa, M. Onga, M. Tokunaga, T. Nojima, Y. Yanase, and Y. Iwasa, Nature Physics 12, 144 EP (2015)

[7] X. Xi, Z. Wang, W. Zhao, J.-H. Park, K. T. Law, H. Berger, L. Forró, J. Shan, and K. F. Mak, Nature Physics 12, 139 EP (2015)

[8] M. M. Ugeda, A. J. Bradley, Y. Zhang, S. Onishi, Y. Chen, W. Ruan, C. Ojeda-Aristizabal, H. Ryu, M. T. Edmonds, H.-Z. Tsai, A. Riss, S.-K. Mo, D. Lee, A. Zettl, Z. Hussain, Z.-X. Shen, and M. F. Crommie, Nature Physics 12, 92 EP (2015)

[9] W. Shi, J. Ye, Y. Zhang, R. Suzuki, M. Yoshida, J. Miyazaki, N. Inoue, Y. Saito, and Y. Iwasa, Scien- 
tific Reports 5, 12534 EP (2015).

[10] S. C. de la Barrera, M. R. Sinko, D. P. Gopalan, N. Sivadas, K. L. Seyler, K. Watanabe, T. Taniguchi, A. W. Tsen, X. Xu, D. Xiao, and B. M. Hunt, Nature Communications 9, 1427 (2018)

[11] E. Navarro-Moratalla, J. O. Island, S. MañasValero, E. Pinilla-Cienfuegos, A. Castellanos-Gomez, J. Quereda, G. Rubio-Bollinger, L. Chirolli, J. A. SilvaGuillén, N. Agraït, G. A. Steele, F. Guinea, H. S. J. van der Zant, and E. Coronado, Nature Communications 7, $11043 \mathrm{EP}$ (2016)

[12] B. T. Zhou, N. F. Q. Yuan, H.-L. Jiang, and K. T. Law, Phys. Rev. B 93, 180501 (2016)

[13] N. F. Q. Yuan, B. T. Zhou, W.-Y. He, and K. T. Law, arXiv:1605.01847 (2016)

[14] E. Sohn, X. Xi, W.-Y. He, S. Jiang, Z. Wang, K. Kang, J.-H. Park, H. Berger, L. Forró, K. T. Law, J. Shan, and K. F. Mak, Nature Materials 17, 504 (2018).

[15] S. Ilić, J. S. Meyer, and M. Houzet, Phys. Rev. Lett. 119, $117001(2017)$

[16] P. A. Frigeri, D. F. Agterberg, A. Koga, and M. Sigrist, Phys. Rev. Lett. 92, 097001 (2004)

[17] W.-Y. He, B. T. Zhou, J. J. He, N. F. Q. Yuan, T. Zhang, and K. T. Law, Communications Physics 1, 40 (2018)

[18] E. Sosenko, J. Zhang, and V. Aji, Phys. Rev. B 95, 144508 (2017)

[19] Y. Nakamura and Y. Yanase, Phys. Rev. B 96, 054501 (2017)

[20] D. Möckli and M. Khodas, Phys. Rev. B 98, 144518 (2018).

[21] D. Möckli and M. Khodas, arXiv:1902.02577

[22] M. H. Fischer, M. Sigrist, and D. F. Agterberg, Phys. Rev. Lett. 121, 157003 (2018)

[23] M. Smidman, M. B. Salamon, H. Q. Yuan, and D. F. Agterberg, Reports on Progress in Physics 80, 036501 (2017)

[24] N. F. Q. Yuan, K. F. Mak, and K. T. Law, Phys. Rev. Lett. 113, 097001 (2014).

[25] R. Oiwa, Y. Yanagi, and H. Kusunose, Phys. Rev. B 98, $064509(2018)$

[26] Y.-T. Hsu, A. Vaezi, M. H. Fischer, and E.-A. Kim, Nature Communications 8, 14985 EP (2017)

[27] L. Wang, T. O. Rosdahl, and D. Sticlet, Phys. Rev. B 98, $205411(2018)$

[28] N. Read and D. Green, Phys. Rev. B 61, 10267 (2000).

[29] R. Oiwa, Y. Yanagi, and H. Kusunose, arXiv:1903.04830

[30] E. Lake, C. Webb, D. A. Pesin, and O. A. Starykh, Phys. Rev. B 93, 214516 (2016)

[31] S. V. Borisenko, A. A. Kordyuk, V. B. Zabolotnyy, D. S. Inosov, D. Evtushinsky, B. Büchner, A. N. Yaresko, A. Varykhalov, R. Follath, W. Eberhardt, L. Patthey, and H. Berger, Phys. Rev. Lett. 102, 166402 (2009)

[32] Y. Nakata, K. Sugawara, S. Ichinokura, Y. Okada, T. Hitosugi, T. Koretsune, K. Ueno, S. Hasegawa, T. Takahashi, and T. Sato, npj 2D Materials and Applications 2, $12(2018)$

[33] A. V. Chubukov, M. Khodas, and R. M. Fernandes, Phys. Rev. X 6, 041045 (2016).

[34] V. Cvetkovic, R. E. Throckmorton, and O. Vafek, Phys. Rev. B 86, 075467 (2012)

[35] M. S. Scheurer and J. Schmalian, Nature Communications 6, $6005 \mathrm{EP}$ (2015)

[36] M. Ye and A. V. Chubukov, Phys. Rev. B 97, 245112
(2018)

[37] P. J. Hirschfeld, M. M. Korshunov, and I. I. Mazin, Reports on Progress in Physics 74, 124508 (2011).

[38] T. V. Trevisan, M. Schütt, and R. M. Fernandes, Phys. Rev. Lett. 121, 127002 (2018)

[39] M. D. Johannes, I. I. Mazin, and C. A. Howells, Phys. Rev. B 73, 205102 (2006)

[40] M. Calandra, I. I. Mazin, and F. Mauri, Phys. Rev. B 80, $241108(2009)$

[41] C. J. Arguello, S. P. Chockalingam, E. P. Rosenthal, L. Zhao, C. Gutiérrez, J. H. Kang, W. C. Chung, R. M. Fernandes, S. Jia, A. J. Millis, R. J. Cava, and A. N. Pasupathy, Phys. Rev. B 89, 235115 (2014)

[42] The relevant functional forms for the gap in each irrep can be found in the spin basis in Ref. 21]; these are related to our gaps by the basis transformation (5).

[43] B. S. Chandrasekhar, Applied Physics Letters 1, 7 (1962).

[44] A. M. Clogston, Phys. Rev. Lett. 9, 266 (1962)

[45] K. V. Samokhin, Phys. Rev. B 92, 174517 (2015).

[46] M. S. Scheurer, D. F. Agterberg, and J. Schmalian, npj Quantum Materials 2, 9 (2017)

[47] D. F. Agterberg, P. M. R. Brydon, and C. Timm, Phys. Rev. Lett. 118, 127001 (2017)

[48] P. M. R. Brydon, D. F. Agterberg, H. Menke, and C. Timm, Phys. Rev. B 98, 224509 (2018)

[49] N. F. Q. Yuan and L. Fu, Phys. Rev. B 97, 115139 (2018)

[50] S. Sumita, T. Nomoto, K. Shiozaki, and Y. Yanase, Phys. Rev. B 99, 134513 (2019)

[51] D. F. Agterberg and R. P. Kaur, Phys. Rev. B 75, 064511 (2007)

[52] C.-X. Liu, Phys. Rev. Lett. 118, 087001 (2017)

[53] A. P. Schnyder and S. Ryu, Phys. Rev. B 84, 060504 (2011)

[54] M. Sato, Y. Tanaka, K. Yada, and T. Yokoyama, Phys. Rev. B 83, 224511 (2011)

[55] S. Matsuura, P.-Y. Chang, A. P. Schnyder, and S. Ryu, New Journal of Physics 15, 065001 (2013)

[56] C.-K. Chiu and A. P. Schnyder, Phys. Rev. B 90, 205136 (2014).

[57] K. Shiozaki and M. Sato, Phys. Rev. B 90, 165114 (2014)

[58] C.-K. Chiu, J. C. Y. Teo, A. P. Schnyder, and S. Ryu, Rev. Mod. Phys. 88, 035005 (2016).

[59] A. Altland and M. R. Zirnbauer, Phys. Rev. B 55, 1142 (1997).

[60] S. Ryu, A. P. Schnyder, A. Furusaki, and A. W. W. Ludwig, New Journal of Physics 12, 065010 (2010)

[61] A. W. W. Ludwig, Physica Scripta T168, 014001 (2015)

[62] In the case of multiple bands, each node changes the $M \mathbb{Z}$ invariant by \pm 1 , depending on which bands cross.

[63] M. Sato and Y. Ando, Reports on Progress in Physics 80, 076501 (2017)

[64] There is also a solution of the gap equation that is even under $p_{x} \rightarrow-p_{x}$, namely the $(x)$ channel, which therefore decouples from the odd solution by symmetry, but which can be shown to always have lower $T_{c}$.

[65] E. Cornfeld and A. Chapman, Phys. Rev. B 99, 075105 (2019)

[66] T. L. Hughes, E. Prodan, and B. A. Bernevig, Phys. Rev. B 83, 245132 (2011)

[67] A. Lau and C. Ortix, The European Physical Journal Special Topics 227, 1309 (2018)

[68] S. Kobayashi, S. Sumita, Y. Yanase, and M. Sato, Phys. Rev. B 97, 180504 (2018) 
[69] E. Witten, 39 (2015).

[70] K. Hashimoto, T. Kimura, and X. Wu, Progress of Theoretical and Experimental Physics $2017 \quad$ (2017), $10.1093 /$ ptep/ptx053 053I01, http://oup.prod.sis.lan/ptep/articlepdf $/ 2017 / 5 / 053 \mathrm{I} 01 / 16637855 /$ ptx053.pdf

[71] G. Bian, T.-R. Chang, H. Zheng, S. Velury, S.-Y. Xu, T. Neupert, C.-K. Chiu, S.-M. Huang, D. S. Sanchez, I. Belopolski, N. Alidoust, P.-J. Chen, G. Chang, A. Bansil, H.-T. Jeng, H. Lin, and M. Z. Hasan, Phys. Rev. B 93, $121113(2016)$

[72] Y.-H. Chan, C.-K. Chiu, M. Y. Chou, and A. P. Schnyder, Phys. Rev. B 93, 205132 (2016)

[73] H. Shapourian, Y. Wang, and S. Ryu, Phys. Rev. B 97, $094508(2018)$

[74] C.-K. Chiu, H. Yao, and S. Ryu, Phys. Rev. B 88, 075142 (2013)

75| C. W. Hicks, D. O. Brodsky, E. A. Yelland, A. S. Gibbs, J. A. N. Bruin, M. E. Barber, S. D. Edkins, K. Nishimura, S. Yonezawa, Y. Maeno, and A. P. Mackenzie, Science 344, 283 (2014)

[76] A. Hamill, B. Heischmidt, E. Sohn, D. Shaffer, K.-T. Tsai, X. Zhang, X. Xi, A. Suslov, H. Berger, L. Forró, F. J. Burnell, J. Shan, K. F. Mak, R. M. Fernandes, K. Wang, and V. S. Pribiag, (2020), arXiv:2004.02999 [cond-mat.supr-con]

[77] C. woo Cho, J. Lyu, T. Han, C. Y. Ng, Y. Gao, G. Li, M. Huang, N. Wang, J. Schmalian, and R. Lortz, (2020), arXiv:2003.12467 [cond-mat.supr-con]

[78] H. Menke, C. Timm, and P. M. R. Brydon, (2019) arXiv:1909.10956

\section{Appendix A: Ginzburg-Landau Free Energy}

Here we write down the Ginzburg-Landau free energy in the presence of SOC and magnetic field. We later

use it in Appendix $\mathrm{B}$ to analyze the chiral phase that emerges in the regime of dominant triplet interactions in the absence of magnetic field and large values of Rashba SOC.

We start with the Bogolyubov-Gor'kov Hamiltonian obtained after doing a Hubbard-Stratonovich transformation:

$$
H=\frac{1}{2} \sum_{\mathbf{p} \eta \tau} \Psi_{\mathbf{p} \eta \tau}^{\dagger} \mathcal{H}_{\eta \tau}(\mathbf{p}) \Psi_{\mathbf{p} \eta \tau}+\frac{1}{2} \sum_{\mathbf{p} \eta \tau} \xi_{\eta \tau}(\mathbf{p})+\mathcal{H}_{0}\left(\Delta^{2}\right)
$$

where

$$
\mathcal{H}_{0}\left(\Delta^{2}\right)=-\frac{1}{4} \sum_{\substack{\mathbf{p} \eta \tau \\ \mathbf{k} \eta^{\prime} \tau^{\prime}}} \Delta_{\eta \tau}^{*}(\mathbf{p})\left(\tilde{V}^{-1}(\mathbf{p} ; \mathbf{k})\right)_{\eta \tau}^{\eta^{\prime} \tau^{\prime}} \Delta_{\eta^{\prime} \tau^{\prime}}(\mathbf{k})
$$

and where we use the Nambu-Gor'kov representation $\Psi_{\eta \tau}(\mathbf{p})=\left(c_{\eta, \mathbf{p} \tau}, c_{-\eta,-\mathbf{p} \tau}^{\dagger}\right)^{T}$ and the BdG Hamiltonian Eq. 47). Recall that the BdG spectrum has two branches, $E_{\eta \tau}(\mathbf{p})$ and, by particle-hole symmetry, $-E_{-\eta \tau}(-\mathbf{p})$. Using the fact that

$$
\operatorname{det}\left[-i \omega+\mathcal{H}_{\eta \tau}(\mathbf{p})\right]=\left(-i \omega+E_{\eta \tau}(\mathbf{p})\right)\left(-i \omega-E_{-\eta \tau}(-\mathbf{p})\right)
$$

we obtain the Ginzburg-Landau free energy:

$$
\mathcal{F}=-\frac{T}{2} \sum_{\mathbf{p} \eta \tau} \ln \left[2 \cosh \left(\frac{\beta E_{\eta \tau}(\mathbf{p})}{2}\right)\right]-\frac{T}{2} \sum_{\mathbf{p} \eta \tau} \ln \left[2 \cosh \left(\frac{\beta E_{-\eta \tau}(-\mathbf{p})}{2}\right)\right]+\mathcal{H}_{0}\left(\Delta^{2}\right)
$$

To obtain the linearized gap equation we expand $\mathcal{F}$ to first order in $\left|\Delta_{\eta \tau}\right|^{2}$, which yields

$$
\mathcal{F}^{(2)}=-\sum_{\mathbf{p} \eta \tau} \frac{\tanh \left(\frac{\beta \xi_{\eta \tau}(\mathbf{p})}{2}\right)+\tanh \left(\frac{\beta \xi_{-\eta \tau}(-\mathbf{p})}{2}\right)}{4 \xi_{S \eta \tau}(\mathbf{p})}\left|\Delta_{\eta \tau}(\mathbf{p})\right|^{2}+\mathcal{H}_{0}\left(\Delta^{2}\right)
$$

Minimizing the free energy Eq. (A5) with respect to $\Delta_{\eta \tau}^{*}(\mathbf{p})$, we obtain the gap equation $(38)$.

\section{Appendix B: Chiral Topological Superconductivity at Large $\alpha_{R}$}

As we discussed in the main text, at zero magnetic field and in the dominant triplet instability regime, at large $\alpha_{R} p_{F}$ the leading superconducting instability is in the two-dimensional $E$ irrep of the point group $C_{3 v}$. Here we show that this state spontaneously breaks time-reversal symmetry (TRS) and compute its Chern number to show that it is a chiral topological state.

\section{Spontaneous Time-Reversal Symmetry Breaking}

We begin by observing that for $b=0$, and assuming equal densities of states on inner and outer Fermi 
surfaces, the reduced gap equation 42 can be solved analytically, giving:

$$
\begin{aligned}
\Delta_{\Gamma \tau}^{(0)}(\mathbf{p}) & =\tau i e^{-i \theta} D_{\Gamma}^{(0)} \\
\Delta_{ \pm K \tau}^{(0)}(\mathbf{p}) & =\tau i e^{-i \theta} D_{K}^{(0)} \\
\Delta_{\Gamma \tau}^{(z)}(\mathbf{p}) & =\sqrt{2} i e^{-i \theta} \cos ^{2} 3 \theta \frac{\lambda p_{F}^{3}}{\delta_{\eta}(\mathbf{p})} D_{\Gamma}^{(z)} \\
\Delta_{ \pm K \tau}^{(z)}(\mathbf{p}) & = \pm i e^{-i \theta} \frac{\beta_{I}}{\delta_{\eta}(\mathbf{p})} D_{K}^{(z)} \\
\Delta_{\Gamma \tau}^{(x)}(\mathbf{p}) & =\sqrt{2} i e^{-i \theta} \sin \theta \cos 3 \theta \frac{\alpha_{R} p_{F}}{\delta_{\eta}(\mathbf{p})} D_{\Gamma}^{(x)} \\
\Delta_{ \pm K \tau}^{(x)}(\mathbf{p}) & = \pm i e^{-i \theta} \sin \theta \frac{\alpha_{R} p_{F}}{\delta_{\eta}(\mathbf{p})} D_{K}^{(x)} \\
\Delta_{\Gamma \tau}^{(y)}(\mathbf{p}) & =\sqrt{2} i e^{-i \theta} \cos \theta \cos 3 \theta \frac{\alpha_{R} p_{F}}{\delta_{\eta}(\mathbf{p})} D_{\Gamma}^{(y)} \\
\Delta_{ \pm K \tau}^{(y)}(\mathbf{p}) & = \pm i e^{-i \theta} \cos \theta \frac{\alpha_{R} p_{F}}{\delta_{\eta}(\mathbf{p})} D_{K}^{(y)}
\end{aligned}
$$

At large $\alpha_{R}$, there is a transition from a mixed $D^{(0)}, D^{(z)}$ solution to a solution with $D^{(0)}=D^{(z)}=0, D^{(x, y)} \neq$ 0 . This solution belongs to the $2 \mathrm{D} E$ irrep of $C_{3 v}$, the relevant point group in this regime. In other words, the $(x)$ and $(y)$ solutions are degenerate, i.e. have the same $T_{c}$.

This degeneracy opens the possibility of spontaneous time-reversal symmetry breaking at $b=0$. In the SOC basis (5), TRS acts as

$$
\begin{gathered}
c_{\eta, \mathbf{p} \tau} \stackrel{\mathcal{T}}{\rightarrow} i \tau e^{i \theta} c_{-\eta,-\mathbf{p} \tau} \\
\Delta_{\eta \tau}(\mathbf{p}) c_{\eta, \mathbf{p} \tau}^{\dagger} c_{-\eta,-\mathbf{p} \tau}^{\dagger} \stackrel{\mathcal{T}}{\rightarrow}-e^{-2 i \theta} \Delta_{\eta \tau}^{*}(\mathbf{p}) c_{\eta, \mathbf{p} \tau}^{\dagger} c_{-\eta,-\mathbf{p} \tau}^{\dagger} .
\end{gathered}
$$

Taking $\Delta_{\eta \tau}(\mathbf{p})=e^{i \Phi_{\eta \tau}(\mathbf{p})}\left|\Delta_{\eta \tau}(\mathbf{p})\right|$, TRS is therefore satisfied when $e^{i \Phi_{\eta \tau}(\mathbf{p})}= \pm i e^{-i \theta}$.

In the linearized gap equation 38 , since the $\mu=x$ and $\mu=y$ channels are degenerate (i.e. they have equal critical temperatures), in principle the relative amplitudes and phases of $D_{\eta}^{(x)}$ and $D_{\eta}^{(y)}$ are not fixed (i.e. any linear combination of the two solutions is allowed). This is no longer the case if we consider the non-linear gap equations. Alternatively, in terms of the Ginzburg-Landau free energy (A4), the relative amplitudes and phases are fixed by the quartic terms in the gap functions. Since $b=0, \xi_{\eta \tau}(-\mathbf{p})=\xi_{\eta \tau}(\mathbf{p})$, the free energy simplifies to

$$
\mathcal{F}=-T \sum_{\mathbf{p} \eta \tau} \ln \left[2 \cosh \left(\frac{\beta E_{\eta \tau}(\mathbf{p})}{2}\right)\right]+\mathcal{H}_{0}\left(\Delta^{2}\right)
$$

Expanding in powers of the gap function, we obtain the fourth order correction (in addition to (A5)):

$$
\mathcal{F}^{(4)}=\frac{7 \zeta(3)}{64 \pi^{2} T^{2}} \sum_{\eta \tau} \int N_{\eta \tau}\left|\Delta_{\eta \tau}(\mathbf{p})\right|^{4} \frac{d \theta_{\mathbf{p}}}{2 \pi}
$$

where $\zeta(x)$ is the Riemann zeta function. Substituting the general form of the gap function in the $(x),(y)$ channel:

$$
\begin{aligned}
\Delta_{\Gamma \tau}(\mathbf{p}) & =\sqrt{2} i e^{-i \theta} \cos 3 \theta \frac{\alpha_{R} p_{F}}{\delta_{\eta}(\mathbf{p})}\left(D_{\Gamma}^{(x)} \cos \theta+D_{\Gamma}^{(y)} \sin \theta\right) \\
\Delta_{ \pm K \tau}(\mathbf{p}) & = \pm i e^{-i \theta} \frac{\alpha_{R} p_{F}}{\delta_{\eta}(\mathbf{p})}\left(D_{K}^{(x)} \cos \theta+D_{K}^{(y)} \sin \theta\right)
\end{aligned}
$$

and approximating $\frac{\alpha_{R} p_{F}}{\delta_{\eta}(\mathbf{p})} \approx 1$ (which is valid as long as $\left.\alpha_{R} p_{F} \gg \lambda p_{F}^{3}\right)$, we obtain:

$$
\begin{aligned}
\mathcal{F}^{(4)} & =\frac{7 \zeta(3)}{2048 \pi^{2} T^{2}} \sum_{\eta \tau} N_{\eta \tau}\left[3\left(\left|D_{\eta}^{(x)}\right|^{2}+\left|D_{\eta}^{(y)}\right|^{2}\right)^{2}\right. \\
& \left.-4\left|D_{\eta}^{(x)}\right|^{2}\left|D_{\eta}^{(y)}\right|^{2} \sin ^{2} \phi_{x y}\right]
\end{aligned}
$$

where $\phi_{x y}$ is the relative phase between $D_{\eta}^{(x)}$ and $D_{\eta}^{(y)}$. Minimization gives $\phi_{x y}= \pm \frac{\pi}{2}$, which implies that the superconducting gap has the form

$$
\begin{aligned}
\Delta_{\Gamma \tau}(\mathbf{p}) & \propto \sqrt{2} i \cos 3 \theta \frac{\alpha_{R} p_{F}}{\delta_{\eta}(\mathbf{p})} e^{-i(\theta \mp \theta)} \\
\Delta_{ \pm K \tau}(\mathbf{p}) & \propto \pm i \frac{\alpha_{R} p_{F}}{\delta_{\eta}(\mathbf{p})} e^{-i(\theta \mp \theta)}
\end{aligned}
$$

which is not invariant under the time-reversal symmetry transformation $(\mathrm{B} 2)$. We thus find that time reversal is spontaneously broken, in agreement with the general result in [45, 46. Note that while $\Delta_{\Gamma \tau}(\mathbf{p})$ obtained from our calculation is nodal, there is an additional symmetry allowed term $\Delta^{(3)}=e^{3 i \theta}$ that belongs to the same $E$ irreducible representation. Adding this term lifts the nodes and results in a fully gapped, time-reversal symmetry broken phase.

\section{Chern Number and Chiral Topological Superconductivity}

To show that this TRS-breaking phase is indeed chiral, we calculate the Chern number, given by

$$
C h=\frac{1}{2 \pi} \sum_{\eta \tau} \int_{B Z} \mathbf{F}_{\eta \tau}(\mathbf{p}) \cdot d^{2} \mathbf{p}
$$

where the Berry curvature vector is given by

$$
\mathbf{F}_{\eta \tau}(\mathbf{p})=\nabla \times \mathbf{A}_{\eta \tau}(\mathbf{p})
$$

with $\mathbf{A}_{\eta \tau}(\mathbf{p})$ the usual Berry connection associated with the occupied band only. For a superconductor, the Berry connection is defined in terms of the normalized eigenvectors of the BdG Hamiltonian (47): $\Upsilon_{\eta \tau}(\mathbf{p})=$ $u_{\eta \tau}(\mathbf{p}) c_{\eta, \mathbf{p} \tau}+v_{\eta \tau}(\mathbf{p}) c_{-\eta,-\mathbf{p} \tau}^{\dagger}$, via

$$
A_{\eta \tau}(\mathbf{p})=i\left\langle\Upsilon_{\eta \tau}(\mathbf{p})\left|\nabla_{\mathbf{p}}\right| \Upsilon_{\eta \tau}(\mathbf{p})\right\rangle
$$

In our case, the $c_{\eta, \mathbf{p} \tau}$ operators may carry a nontrivial Berry phase due to the changing orientation of the associated spin. One should therefore consider $\left|\Upsilon_{\eta \tau}(\mathbf{p})\right\rangle$ as a 
four component eigenvector in a basis of Nambu-Gor'kov 4-spinors $\Psi_{\eta \tau}^{(4)}(\mathbf{p})=\left(d_{\eta, \mathbf{p} \uparrow}, d_{\eta, \mathbf{p} \downarrow}, d_{-\eta,-\mathbf{p} \uparrow}^{\dagger}, d_{-\eta,-\mathbf{p} \downarrow}^{\dagger}\right)^{T}$. Using the change of basis (5), we find

$$
\left|\Upsilon_{\eta \tau}\right\rangle=\left(\begin{array}{c}
U_{\eta \tau}^{1}(\mathbf{p}) u_{\eta \tau}(\mathbf{p}) \\
U_{\eta \tau}^{-1}(\mathbf{p}) u_{\eta \tau}(\mathbf{p}) \\
U_{-\eta \tau}^{1 *}(-\mathbf{p}) v_{\eta \tau}(\mathbf{p}) \\
U_{-\eta \tau}^{-1 *}(-\mathbf{p}) v_{\eta \tau}(\mathbf{p})
\end{array}\right)
$$

Thus

$$
\begin{aligned}
& u_{\eta \tau}(\mathbf{p})=\frac{\xi_{S \eta \tau}-E_{\eta \tau}(\mathbf{p})}{\sqrt{\left(\xi_{S \eta \tau}-E_{\eta \tau}(\mathbf{p})\right)^{2}+\left|\Delta_{\eta \tau}(\mathbf{p})\right|^{2}}} \\
& v_{\eta \tau}(\mathbf{p})=\frac{\Delta_{\eta \tau}(\mathbf{p})}{\sqrt{\left(\xi_{S \eta \tau}-E_{\eta \tau}(\mathbf{p})\right)^{2}+\left|\Delta_{\eta \tau}(\mathbf{p})\right|^{2}}}
\end{aligned}
$$

where we use the notation of Eq. 48,50.

Below we calculate the Chern number for $b=$ 0 and non-zero $\alpha_{R}$ only, in which case $U_{\eta \tau}^{1}(\mathbf{p})=$ $-i\left|U_{\eta \tau}^{1}(\mathbf{p})\right| e^{-i \theta_{\eta, \mathbf{p}}}$ where $\theta_{\eta, \mathbf{p}}$ is the angle of the momentum $\mathbf{p}$ measured relative to the center of the Fermi pocket $\eta$. Defining as before $\Delta_{\eta \tau}(\mathbf{p})=\left|\Delta_{\eta \tau}(\mathbf{p})\right| e^{i \Phi_{\eta \tau}(\mathbf{p})}$, we find that in this regime the Berry connection associated with the pocket $\eta$ is

$$
A_{\eta \tau}(\mathbf{p})=\left|U_{\eta \tau}^{1}(\mathbf{p})\right|^{2} \nabla \theta_{\eta \mathbf{p}}-\left|v_{\eta \tau}(\mathbf{p})\right|^{2}\left(\nabla \Phi_{\eta \tau}(\mathbf{p})+\nabla \theta_{\eta \mathbf{p}}\right)
$$

For the two TRS-breaking gaps given in Eq. (B6), we have $\Phi_{\eta \tau}=0$ and $\Phi_{\eta \tau}=-2 \theta_{\eta}$, respectively.

To obtain the Chern number we insert these expressions into (B13), and integrate over an annulus around each component of the Fermi surface. To evaluate this integral, we assume with no loss of generality that the gap function is constant in some region around the FS, and completely vanishing in regions sufficiently far from the FS, with a phase independent of the radial direction $p$, and take $\left|U_{\eta \tau}^{1}(\mathbf{p})\right|$ to be independent of $p$. Finally, observe that $v_{\eta \tau}$ changes rapidly from 0 to 1 in the vicinity of the Fermi surface. For the pocket $\eta$, we therefore obtain:

$$
\begin{array}{r}
C h_{\eta}=\frac{1}{2 \pi} \int\left(F_{\eta \tau}(\mathbf{p})\right)_{p \theta} d p d \theta=\frac{1}{2 \pi} \int \partial_{p}\left(A_{\eta \tau}(\mathbf{p})\right)_{\theta} d p d \theta \\
=\frac{1}{2 \pi}\left[\int\left(A_{\eta \tau}(\mathbf{p})\right)_{\theta} d \theta\right]_{p=0}^{p=\infty}=-\frac{1}{2 \pi}\left[\Phi_{\eta \tau}(\mathbf{p})+\theta_{\eta, \mathbf{p}}\right]_{0}^{2 \pi}
\end{array}
$$

where the integrals over $\theta$ and $p$ are understood to be over the tangential and normal directions in a disk including the Fermi surface of the $\eta$ pocket, respectively. This gives a net Chern number of \pm 6 , with a total contribution of \pm 4 from the $\pm K$ pockets, and of \pm 2 from the $\Gamma$ pocket.

We emphasize that this result is independent of the choice of $\Delta_{\eta \tau}$ away from the Fermi surface. This is because only the region proximate to the Fermi surface contributes to the integral in Eq. (B7). To see this, notice that any two choices of the superconducting gap away from the Fermi surface must yield the same result, as a topological phase transition requires band touching that can only occur when $\xi_{S \eta \tau}$ and $\Delta_{\eta \tau}$ are simultaneously zero in Eq. (48) (as can be directly verified by constructing a simple homotopy between Hamiltonians with any two such choices).

\section{Appendix C: Tight-binding model on a cylinder}

To study the edge modes in more detail and produce the plot in Fig. 6 we used a tight binding model defined on the triangular lattice. The Hamiltonian has the general form

$$
H=H_{0}+H_{Z}+H_{S C}
$$

The first term describes the normal state band structure in the presence of SOC; the second-term is the Zeeman coupling due to in-plane magnetic field; and the last term represents the superconducting pairing gap. For simplicity we use a tight-binding model that only includes the $\eta=\Gamma$ pocket Fermi surface, since the $\pm K$ pockets are unimportant for the crystalline nodal topological superconductor.

We describe our model in terms of the creation operators $d_{i, \alpha}^{\dagger}$, where $\alpha=\uparrow, \downarrow$ is a spin index, and $i$ is a site index. We have

$$
\begin{aligned}
H_{0}= & \sum_{i \alpha} \mu d_{i \alpha}^{\dagger} d_{i \alpha}+\sum_{\langle i j\rangle \alpha} t d_{i \alpha}^{\dagger} d_{j \alpha} \\
& +\sum_{\langle i j\rangle \alpha \beta}\left[4 i \lambda \nu_{i j} \sigma_{\alpha \beta}^{z}+\frac{i \alpha_{R}}{3} \hat{\mathbf{z}} \cdot\left(\boldsymbol{\sigma} \times \mathbf{a}_{i j}\right)_{\alpha \beta}\right] d_{i \alpha}^{\dagger} d_{j \beta} \\
H_{Z}= & \sum_{i \alpha \beta}(\mathbf{b} \cdot \boldsymbol{\sigma})_{\alpha \beta} d_{i \alpha}^{\dagger} d_{i \beta} \\
H_{S C}= & \frac{1}{2} \sum_{i j \alpha \beta}[\Delta]_{\alpha \beta}^{i j} d_{i \alpha}^{\dagger} d_{j \beta}^{\dagger}+\text { h.c. }
\end{aligned}
$$

where $\mathbf{a}_{i j} \in\left\{ \pm \mathbf{a}_{1}, \pm \mathbf{a}_{2}, \pm \mathbf{a}_{3}\right\}$ is the vector from site $i$ to site $j$, and $\nu_{i j}=1(-1)$ if the vector is $\mathbf{a}_{1},-\mathbf{a}_{2}, \mathbf{a}_{3}$ ( $\left.-\mathbf{a}_{1}, \mathbf{a}_{2},-\mathbf{a}_{3}\right)$. For our triangular lattice, $\mathbf{a}_{1}=(a, 0)$ and $\mathbf{a}_{2}=\frac{a}{2}(1, \sqrt{3}), \mathbf{a}_{3}=\mathbf{a}_{2}-\mathbf{a}_{1}=\frac{a}{2}(-1, \sqrt{3})$. We consider the singlet-instability regime, in the crystalline nodal topological phase where $b \gg \alpha_{R}$. In this region the self-consistent solutions of the gap equation obtained in a $\mathbf{k} \cdot \mathbf{p}$ model are well-approximated by

$$
\Delta^{i j}=\Delta_{t} \nu_{i j}\left(\sigma^{x} \cos \vartheta+\sigma^{y} \sin \vartheta\right) i \sigma^{y}+\Delta_{s} i \sigma^{y}
$$

where $\vartheta$ is the direction of the magnetic field, assuming $\Delta_{s} \ll \Delta_{t}$ (higher lattice harmonics are in general needed to match the $\mathbf{k} \cdot \mathbf{p}$ model exactly). The numerical coefficients are chosen to match the $\mathbf{k} \cdot \mathbf{p}$ Hamiltonian (including the value of $p_{F}$ ).

Our cylinder is created by taking periodic boundary conditions in the vertical $y$ direction, and open zig-zag 
boundary conditions along the $x$ direction. To produce the plot, we Fourier transform in the $y$ direction:

$d_{\mathbf{R}_{\mathbf{i}} \alpha}=\frac{1}{\sqrt{N}} \sum_{p_{y}} d_{R_{i x} p_{y} \alpha} e^{-i p_{y} R_{i y}} \equiv \frac{1}{\sqrt{N}} \sum_{p_{y}} d_{i p_{y} \alpha} e^{-i p_{y} R_{i y}}$

where $\mathbf{R}_{i}=\left(R_{i x}, R_{i y}\right)$. Note that $i$ labels the $x$ coordinates of the sites which go in increments of $a / 2$, while the period along the $y$ axis is actually doubled since identical sites are now separated by $2 \mathbf{a}_{2}$, resulting in the folding of the 1D Brillouin zone (which has a period of $\frac{2 \pi}{\sqrt{3} a}$ ).

The resulting BdG Hamiltonian on the cylinder can be expressed

$$
H_{B d G}=\frac{1}{2} \sum_{i j, p_{y}} \Psi_{i, p_{y}}^{\dagger} \mathcal{H}^{i j}\left(p_{y}\right) \Psi_{j,-p_{y}}
$$

where $\Psi_{i, p_{y}}=\left(d_{i, p_{y} \uparrow}, d_{i, p_{y} \downarrow}, d_{i,-p_{y} \uparrow}^{\dagger}, d_{i,-p_{y} \downarrow}^{\dagger}\right)$ and

$$
\mathcal{H}^{i j}\left(p_{y}\right)=\left(\begin{array}{cc}
\mathcal{H}_{k i n}^{i j}\left(p_{y}\right) & \Delta^{i j}\left(p_{y}\right) \\
-\left(\Delta^{i j}\left(-p_{y}\right)\right)^{*} & -\left(\mathcal{H}_{k i n}^{T}\left(-p_{y}\right)\right)^{j i}
\end{array}\right)
$$

where we have defined

$$
H_{0}+H_{Z}=\sum_{i j \alpha \beta}\left(\mathcal{H}_{k i n}\left(p_{y}\right)\right)_{\alpha \beta}^{i j} d_{i p_{y} \alpha}^{\dagger} d_{j p_{y} \beta}
$$

In Fig. 6we plot the spectrum of (C6) with the number of sites along the non-periodic $x$ direction $N=300$ (which corresponds to 150 unit cells due to period doubling). We also set $t=1, \mu=0, \beta_{I}=1, \lambda=0.2, b=1, \alpha_{R}=0.1$, $\Delta_{t}=1$ and $\Delta_{s}=0.1$. The magnetic field was again aligned along one of the $\Gamma-K$ directions, $\vartheta=0$. 